\title{
Local symplectic algebra of quasi-homogeneous curves
}

by

\author{
Wojciech Domitrz (Warszawa)
}

\begin{abstract}
We study the local symplectic algebra of parameterized curves introduced by V. I. Arnold. We use the method of algebraic restrictions to classify symplectic singularities of quasi-homogeneous curves. We prove that the space of algebraic restrictions of closed 2-forms to the germ of a $\mathbb{K}$-analytic curve is a finite-dimensional vector space. We also show that the action of local diffeomorphisms preserving the quasi-homogeneous curve on this vector space is determined by the infinitesimal action of liftable vector fields. We apply these results to obtain a complete symplectic classification of curves with semigroups $(3,4,5),(3,5,7),(3,7,8)$.
\end{abstract}

1. Introduction. We study the problem of classification of parameterized curve-germs in a symplectic space $\left(\mathbb{K}^{2 n}, \omega\right)$ up to symplectic equivalence (for $\mathbb{K}=\mathbb{R}$ or $\mathbb{C}$ ). The symplectic equivalence is a right-left equivalence (or $\mathcal{A}$-equivalence) in which the left diffeomorphism-germ is a symplectomorphism of $\left(\mathbb{K}^{2 n}, \omega\right)$, i.e. it preserves the given symplectic form $\omega$ in $\mathbb{K}^{2 n}$.

The problem of $\mathcal{A}$-classification of singularities of parameterized curvegerms was studied by J. W. Bruce and T. J. Gaffney [BG] and C. G. Gibbson and C. A. Hobbs $[\mathrm{GH}]$ who classified the $\mathcal{A}$-simple plane curves and the $\mathcal{A}$-simple space curves respectively. A singularity (an $\mathcal{A}$-equivalence class) is called simple if it has a neighborhood intersecting only a finite number of singularities. V. I. Arnold [A2] classified stably simple singularities of curves. A singularity is stably simple if it is simple and remains simple after embedding into a larger space.

The main tool and the invariant separating the singularities in the $\mathcal{A}$ classification of curves is the semigroup of a curve singularity $t \mapsto f(t)=$ $\left(f_{1}(t), \ldots, f_{m}(t)\right)$ (see $[\mathrm{GH}]$ and $\left.[\mathrm{A} 2]\right)$. It is the subsemigroup of the additive semigroup of natural numbers formed by the orders of zero at the origin of all linear combinations of products of $f_{i}(t)$.

2000 Mathematics Subject Classification: Primary 53D05; Secondary 14H20, 58K50, $58 \mathrm{~A} 10$.

Key words and phrases: symplectic manifold, curves, local symplectic algebra, algebraic restrictions, relative Darboux theorem, singularities. 
In [A1] V. I. Arnold discovered new symplectic invariants of parameterized curves. He proved that the $A_{2 k}$ singularity of a planar curve (the orbit with respect to standard $\mathcal{A}$-equivalence of parameterized curves) splits into exactly $2 k+1$ symplectic singularities (orbits with respect to symplectic equivalence of parameterized curves). Arnold posed the problem of expressing these invariants in terms of the local algebra's interaction with the symplectic structure. He proposed to call this interaction local symplectic algebra.

In [IJ1] G. Ishikawa and S. Janeczko classified symplectic singularities of curves in the 2-dimensional symplectic space. All simple curves in this classification are quasi-homogeneous (see also $[\mathrm{DR}]$ ).

A symplectic singularity is stably simple if it is simple and remains simple if the ambient symplectic space is symplectically embedded (i.e. as a symplectic submanifold) into a larger symplectic space. In [K] P. A. Kolgushkin classified the stably simple symplectic singularities of curves (in the $\mathbb{C}$-analytic category). All stably simple symplectic singularities of curves are also quasi-homogeneous.

In [DJZ2] new symplectic invariants of singular quasi-homogeneous subsets of a symplectic space were described by the algebraic restrictions of the symplectic form to these subsets.

The algebraic restriction is an equivalence class of the following relation on the space of differential $k$-forms:

Differential $k$-forms $\omega_{1}$ and $\omega_{2}$ have the same algebraic restriction to a subset $N$ if $\omega_{1}-\omega_{2}=\alpha+d \beta$, where $\alpha$ is a $k$-form vanishing on $N$ and $\beta$ is a $(k-1)$-form vanishing on $N$.

The algebraic restriction of a $k$-form $\omega_{1}$ to a subset $N_{1}$ and the algebraic restriction of a $k$-form $\omega_{2}$ to a subset $N_{2}$ are diffeomorphic if there exists a diffeomorphism $\Phi$ of $\mathbb{K}^{m}$ which maps $N_{1}$ to $N_{2}$ such that $\Phi^{*} \omega_{2}$ and $\omega_{1}$ have the same algebraic restriction to $N_{1}$ (for details see Section 3 ).

The results in [DJZ2] were obtained by the following generalization of the Darboux-Givental theorem.

THEOREM 1 ([DJZ2]). Quasi-homogeneous subsets of a symplectic manifold $(M, \omega)$ are locally symplectomorphic if and only if the algebraic restrictions of the symplectic form $\omega$ to these subsets are locally diffeomorphic.

This theorem reduces the problem of symplectic classification of quasihomogeneous subsets to the problem of classification of algebraic restrictions of symplectic forms to these subsets.

In [DJZ2] the method of algebraic restrictions is applied to various classification problems in a symplectic space. In particular, a complete symplectic classification of classical A-D-E singularities of planar curves is obtained, which contains Arnold's symplectic classification of the $A_{2 k}$ singularity. 
In this paper we return to Arnold's original problem of local symplectic algebra of a parameterized curve. We show that the method of algebraic restrictions is a very powerful classification tool for quasi-homogeneous parameterized curves. This is due to the several reasons. The most important one is that the space of algebraic restrictions of germs of closed 2-forms to a $\mathbb{K}$-analytic parameterized curve is a finite-dimensional vector space. This fact follows from the following more general result conjectured in [DJZ2], which we prove in this paper.

TheOREM 2. Let $C$ be the germ of a $\mathbb{K}$-analytic curve. Then the space of algebraic restrictions to $C$ of germs of closed 2 -forms is a finite-dimensional vector space.

By a $\mathbb{K}$-analytic curve we understand a subset of $\mathbb{K}^{m}$ which is locally diffeomorphic to a 1-dimensional (possibly singular) $\mathbb{K}$-analytic subvariety of $\mathbb{K}^{m}$. Germs of $\mathbb{C}$-analytic parameterized curves can be identified with germs of irreducible $\mathbb{C}$-analytic curves.

The tangent space to the orbit of an algebraic restriction $a$ to the germ $f$ of a parameterized curve is given by the Lie derivative of $a$ with respect to germs of liftable vector fields over $f$. We say that the germ $X$ of a liftable vector field acts trivially on the space of algebraic restrictions if the Lie derivative of any algebraic restriction with respect to $X$ is zero.

TheOREM 3. The space of germs of liftable vector fields over the germ of a parameterized quasi-homogeneous curve which act non-trivially on the space of algebraic restrictions of closed 2 -forms is a finite-dimensional vector space.

Theorem 2 is proved in Section 5. In Section 6 we prove Theorem 3 using the quasi-homogeneous grading on the space of algebraic restrictions. We show that there exist quasi-homogeneous bases of the space of algebraic restrictions of closed 2-forms and of the space of liftable vector fields which act non-trivially on the space of algebraic restrictions to a quasi-homogeneous parameterized curve. These bases allow us to prove Theorem 6.13 that states that the linear action on the space of algebraic restrictions of closed 2-forms to the germ of a quasi-homogeneous parameterized curve by Lie derivatives with respect to liftable vector fields determines the action on this space by local diffeomorphisms preserving this germ of the curve.

Both the space of algebraic restrictions of symplectic forms and this linear action are determined by the semigroup of the curve singularity.

We apply the method of algebraic restrictions and results of Section 6 to obtain a complete symplectic classification of curves with semigroups $(3,4,5),(3,5,7)$ and $(3,7,8)$ in Sections 7,8 and 9 . The classification results 
are presented in Tables 1, 5 and 9. All normal forms are given in the canonical coordinates $\left(p_{1}, q_{1}, \ldots, p_{n}, q_{n}\right)$ in the symplectic space $\left(\mathbb{R}^{2 n}, \sum_{i=1}^{n} d p_{i} \wedge d q_{i}\right)$. The parameters $c, c_{1}, c_{2}$ are moduli. The different singularity classes are distinguished by discrete symplectic invariants: the symplectic multiplicity $\mu_{\text {sympl }}(f)$, the index of isotropy $i(f)$ and the Lagrangian tangency order Lt $(f)$, which are considered in Section 4.

We consider only quasi-homogeneous parameterized curves in this paper. But there are $\mathcal{A}$-simple singularities of curves which are not quasihomogeneous. For example, the curve $f(t)=\left(t^{3}, t^{7}+t^{8}\right)$ is not quasi-homogeneous. Theorem 1 cannot be applied to such curves. But there exists a generalization of this theorem to any subsets $N$ of $\mathbb{K}^{m}$ ([DJZ2, Section $2.6])$. In general there is one more invariant for the symplectic classification problem which can be represented by a cohomology class in the second cohomology group of the complex of 2 -forms with zero algebraic restrictions to $N$. These cohomology groups vanish for quasi-homogeneous subsets ([DJZ1]). They are finite-dimensional for $\mathbb{C}$-analytic varieties with an isolated singularity $([\mathrm{BH}])$. This implies that they are finite-dimensional for non-quasi-homogeneous $\mathbb{C}$-analytic curves. The space of algebraic restrictions of closed 2 -forms to a $\mathbb{K}$-analytic curve is also finite-dimensional by Theorem 2. But the description of the action on algebraic restrictions of diffeomorphisms preserving a non-quasi-homogeneous curve is much more complicated.

2. Quasi-homogeneity. In this section we present the basic definitions and properties of quasi-homogeneous germs.

Definition 2.1. A curve-germ $f:(\mathbb{K}, 0) \rightarrow\left(\mathbb{K}^{m}, 0\right)$ is quasi-homogeneous if there exist coordinate systems $t$ on $(\mathbb{K}, 0)$ and $\left(x_{1}, \ldots, x_{m}\right)$ on $\left(\mathbb{K}^{m}, 0\right)$ and positive integers $\left(\lambda_{1}, \ldots, \lambda_{m}\right)$ such that

$$
d f\left(t \frac{d}{d t}\right)=E \circ f
$$

where $E=\sum_{i=1}^{m} \lambda_{i} x_{i} \partial / \partial x_{i}$ is the germ of the Euler vector field on $\left(\mathbb{K}^{m}, 0\right)$. The coordinate system $\left(x_{1}, \ldots, x_{m}\right)$ is then also called quasi-homogeneous, and the numbers $\left(\lambda_{1}, \ldots, \lambda_{m}\right)$ are called weights.

Definition 2.2. Positive integers $\lambda_{1}, \ldots, \lambda_{m}$ are linearly dependent over non-negative integers if there exists $j$ and non-negative integers $k_{i}$ for $i \neq j$ such that $\lambda_{j}=\sum_{i \neq j} k_{i} \lambda_{i}$. Otherwise we say that $\lambda_{1}, \ldots, \lambda_{m}$ are linearly independent over non-negative integers.

It is easy to see that quasi-homogeneous curves have the following form in quasi-homogeneous coordinates. 
Proposition 2.3. A curve-germ $f$ is quasi-homogeneous if and only if $f$ is $\mathcal{A}$-equivalent to

$$
t \mapsto\left(t^{\lambda_{1}}, \ldots, t^{\lambda_{k}}, 0, \ldots, 0\right),
$$

where $\lambda_{1}<\cdots<\lambda_{k}$ are positive integers linearly independent over nonnegative integers.

$\lambda_{1}, \ldots, \lambda_{k}$ generate the semigroup of the curve $f$, which we denote by $\left(\lambda_{1}, \ldots, \lambda_{k}\right)$.

The weights $\lambda_{1}, \ldots, \lambda_{k}$ are determined by $f$, but the weights $\lambda_{k+1}, \ldots, \lambda_{m}$ can be arbitrary positive integers. Actually in the next sections we study the projection of $f$ to the non-zero components: $\mathbb{K} \ni t \mapsto\left(t^{\lambda_{1}}, \ldots, t^{\lambda_{k}}\right) \in \mathbb{K}^{k}$.

Definition 2.4. The germ of a function, of a differential $k$-form, or of a vector field $\alpha$ on $\left(\mathbb{K}^{m}, 0\right)$ is quasi-homogeneous in a coordinate system $\left(x_{1}, \ldots, x_{m}\right)$ on $\left(\mathbb{K}^{m}, 0\right)$ with positive weights $\left(\lambda_{1}, \ldots, \lambda_{m}\right)$ if $\mathcal{L}_{E} \alpha=\delta \alpha$, where $E=\sum_{i=1}^{m} \lambda_{i} x_{i} \partial / \partial x_{i}$ is the germ of the Euler vector field on $\left(\mathbb{K}^{m}, 0\right)$ and $\delta$ is a real number called the quasi-degree.

It is easy to show that $\alpha$ is quasi-homogeneous in a coordinate system $\left(x_{1}, \ldots, x_{m}\right)$ with weights $\left(\lambda_{1}, \ldots, \lambda_{m}\right)$ if and only if $F_{t}^{*} \alpha=t^{\delta} \alpha$, where $F_{t}\left(x_{1}, \ldots, x_{m}\right)=\left(t^{\lambda_{1}} x_{1}, \ldots, t^{\lambda_{m}} x_{m}\right)$. Thus germs of quasi-homogeneous functions of quasi-degree $\delta$ are germs of weighted homogeneous polynomials of degree $\delta$. The coefficient $f_{i_{1}, \ldots, i_{k}}$ of the quasi-homogeneous differential $k$-form $\sum f_{i_{1}, \ldots, i_{k}} d x_{i_{1}} \wedge \cdots \wedge d x_{i_{k}}$ of quasi-degree $\delta$ is a weighted homogeneous polynomial of degree $\delta-\sum_{j=1}^{k} \lambda_{i_{j}}$. The coefficient $f_{i}$ of the quasi-homogeneous vector field $\sum_{i=1}^{m} f_{i} \partial / \partial x_{i}$ of quasi-degree $\delta$ is a weighted homogeneous polynomial of degree $\delta+\lambda_{i}$.

Proposition 2.5. If $X$ is the germ of a quasi-homogeneous vector field of quasi-degree $i$ and $\omega$ is the germ of a quasi-homogeneous differential form of quasi-degree $j$ then $\mathcal{L}_{X} \omega$ is the germ of a quasi-homogeneous differential form of quasi-degree $i+j$.

Proof. Since $\mathcal{L}_{E} X=[E, X]=i X$ and $\mathcal{L}_{E} \omega=j \omega$, we have

$$
\begin{aligned}
\mathcal{L}_{E}\left(\mathcal{L}_{X} \omega\right) & =\mathcal{L}_{X}\left(\mathcal{L}_{E} \omega\right)+\mathcal{L}_{[E, X]} \omega=\mathcal{L}_{X}(j \omega)+\mathcal{L}_{i X} \omega \\
& =j \mathcal{L}_{X} \omega+i \mathcal{L}_{X} \omega=(i+j) \mathcal{L}_{X} \omega .
\end{aligned}
$$

This implies that $\mathcal{L}_{X} a$ is quasi-homogeneous of quasi-degree $i+j$.

3. The method of algebraic restrictions. In this section we present basic facts on the method of algebraic restrictions. The proofs can be found in [DJZ2].

Given the germ of a smooth manifold $(M, p)$ denote by $\Lambda^{k}(M)$ the space of all germs at $p$ of differential $k$-forms on $M$. Given a curve-germ $f$ : 
$(\mathbb{K}, 0) \rightarrow(M, p)$ introduce the following subspaces of $\Lambda^{p}(M)$ :

$$
\begin{aligned}
\Lambda_{\operatorname{Im} f}^{p}(M) & =\left\{\omega \in \Lambda^{p}(M):\left.\omega\right|_{f(t)}=0 \text { for any } t \in \mathbb{K}\right\}, \\
\mathcal{A}_{0}^{p}(\operatorname{Im} f, M) & =\left\{\alpha+d \beta: \alpha \in \Lambda_{\operatorname{Im} f}^{p}(M), \beta \in \Lambda_{\operatorname{Im} f}^{p-1}(M)\right\} .
\end{aligned}
$$

The relation $\left.\omega\right|_{f(t)}=0$ means that the $p$-form $\omega$ annihilates any $p$-tuple of vectors in $T_{f(t)} M$, i.e. all coefficients of $\omega$ in some (and then any) local coordinate system vanish at the point $f(t)$.

DeFINITION 3.1. The algebraic restriction of $\omega$ to a curve-germ $f: \mathbb{K} \rightarrow$ $M$ is the equivalence class of $\omega$ in $\Lambda^{p}(M)$, where $\omega$ is equivalent to $\widetilde{\omega}$ if $\omega-\widetilde{\omega} \in \mathcal{A}_{0}^{p}(\operatorname{Im} f, M)$.

Notation. The algebraic restriction of the germ of a form $\omega$ on $(M, p)$ to a curve-germ $f$ will be denoted by $[\omega]_{f}$. Writing $[\omega]_{f}=0$ (or saying that $\omega$ has zero algebraic restriction to $f)$ we mean that $[\omega]_{f}=[0]_{f}$, i.e. $\omega \in \mathcal{A}_{0}^{p}(\operatorname{Im} f, M)$.

REMARK 3.2. If $g=f \circ \phi$ for a local diffeomorphism $\phi$ of $\mathbb{K}$ then the algebraic restrictions $[\omega]_{f}$ and $[\omega]_{g}$ can be identified, because $\operatorname{Im} f=\operatorname{Im} g$.

Let $(M, p)$ and $(\widetilde{M}, \tilde{p})$ be germs of smooth equi-dimensional manifolds. Let $f:(\mathbb{K}, 0) \rightarrow(M, p)$ be a curve-germ in $(M, p)$. Let $\tilde{f}:(\mathbb{K}, 0) \rightarrow(\widetilde{M}, \tilde{p})$ be a curve-germ in $(\widetilde{M}, \tilde{p})$. Let $\omega$ be the germ of a $k$-form on $(M, p)$ and $\widetilde{\omega}$ be the germ of a $k$-form on $(\widetilde{M}, \tilde{p})$.

Definition 3.3. Algebraic restrictions $[\omega]_{f}$ and $[\widetilde{\omega}]_{\tilde{f}}$ are called diffeomorphic if there exists a germ of a diffeomorphism $\Phi:(\widetilde{M}, \tilde{p}) \rightarrow(M, p)$ and a germ of a diffeomorphism $\phi:(\mathbb{K}, 0) \rightarrow(\mathbb{K}, 0)$ such that $\Phi \circ \tilde{f} \circ \phi=f$ and $\Phi^{*}\left([\omega]_{f}\right):=\left[\Phi^{*} \omega\right]_{\Phi^{-1} \circ f}=[\widetilde{\omega}]_{\tilde{f}}$.

REMARK 3.4. The above definition does not depend on the choice of $\omega$ and $\widetilde{\omega}$ since a local diffeomorphism maps forms with zero algebraic restriction to $f$ to forms with zero algebraic restriction to $\tilde{f}$. If $(M, p)=(\widetilde{M}, \tilde{p})$ and $f=\tilde{f}$ then the definition of diffeomorphic algebraic restrictions reduces to the following one: two algebraic restrictions $[\omega]_{f}$ and $[\widetilde{\omega}]_{f}$ are diffeomorphic if there exist germs of diffeomorphisms $\Phi$ of $(M, p)$ and $\phi$ of $(\mathbb{K}, 0)$ such that $\Phi \circ f \circ \phi=f$ and $\left[\Phi^{*} \omega\right]_{f}=[\widetilde{\omega}]_{f}$.

The method of algebraic restrictions applied to singular quasi-homogeneous curves is based on the following theorem.

Theorem 3.5 (Theorem A in [DJZ2]). Let $f:(\mathbb{K}, 0) \rightarrow\left(\mathbb{K}^{2 n}, 0\right)$ be the germ of a quasi-homogeneous curve. If $\omega_{0}, \omega_{1}$ are germs of symplectic forms on $\left(\mathbb{K}^{2 n}, 0\right)$ with the same algebraic restriction to $f$ then there exists a germ of a diffeomorphism $\Phi:\left(\mathbb{K}^{2 n}, 0\right) \rightarrow\left(\mathbb{K}^{2 n}, 0\right)$ such that $\Phi \circ f=f$ and $\Phi^{*} \omega_{1}=\omega_{0}$. 
Two germs of quasi-homogeneous curves $f, g$ of a fixed symplectic space $\left(\mathbb{K}^{2 n}, \omega\right)$ are symplectically equivalent if and only if the algebraic restrictions of the symplectic form $\omega$ to $f$ and $g$ are diffeomorphic.

Theorem 3.5 reduces the problem of symplectic classification of singular quasi-homogeneous curves to the problem of diffeomorphic classification of algebraic restrictions of symplectic forms to a singular quasi-homogeneous curve.

In Section 5 we prove that the set of algebraic restrictions of 2-forms to a $\mathbb{K}$-analytic curve is a finite-dimensional vector space. We now recall the basic properties of algebraic restrictions which are useful for a description of this subset [DJZ2].

Let $f$ be a quasi-homogeneous curve on $\left(\mathbb{K}^{2 n}, 0\right)$.

First we can reduce the dimension of the manifold we consider due to the following propositions.

Proposition 3.6. Let $(M, 0)$ be the germ of a smooth submanifold of $\left(\mathbb{K}^{m}, 0\right)$ containing $\operatorname{Im} f$. Let $\omega_{1}, \omega_{2}$ be germs of $k$-forms on $\left(\mathbb{K}^{m}, 0\right)$. Then $\left[\omega_{1}\right]_{f}=\left[\omega_{2}\right]_{f}$ if and only if $\left[\left.\omega_{1}\right|_{T M}\right]_{f}=\left[\left.\omega_{2}\right|_{T M}\right]_{f}$.

Proposition 3.7. Let $f_{1}, f_{2}$ be curve-germs in $\left(\mathbb{K}^{m}, 0\right)$ whose images are contained in germs of equi-dimensional smooth submanifolds $\left(M_{1}, 0\right)$, $\left(M_{2}, 0\right)$ respectively. Let $\omega_{1}, \omega_{2}$ be germs of $k$-forms on $\left(\mathbb{K}^{m}, 0\right)$. The algebraic restrictions $\left[\omega_{1}\right]_{f_{1}}$ and $\left[\omega_{2}\right]_{f_{2}}$ are diffeomorphic if and only if the algebraic restrictions $\left[\left.\omega_{1}\right|_{T M_{1}}\right]_{f_{1}}$ and $\left[\left.\omega_{2}\right|_{T M_{2}}\right]_{f_{2}}$ are diffeomorphic.

To calculate the space of algebraic restrictions of 2 -forms we will use the following obvious properties.

Proposition 3.8. If $\omega \in \mathcal{A}_{0}^{k}\left(\operatorname{Im} f, \mathbb{K}^{2 n}\right)$ then $d \omega \in \mathcal{A}_{0}^{k+1}\left(\operatorname{Im} f, \mathbb{K}^{2 n}\right)$ and $\omega \wedge \alpha \in \mathcal{A}_{0}^{k+p}\left(\operatorname{Im} f, \mathbb{K}^{2 n}\right)$ for any $p$-form $\alpha$ on $\mathbb{K}^{2 n}$.

The next step of our calculation is the description of the subspace of algebraic restrictions of closed 2 -forms. The following proposition is very useful for this step.

Proposition 3.9. Let $a_{1}, \ldots, a_{k}$ be a basis of the space of algebraic restrictions of 2-forms to $f$ satisfying the following conditions:

(1) $d a_{1}=\cdots=d a_{j}=0$

(2) the algebraic restrictions $d a_{j+1}, \ldots, d a_{k}$ are linearly independent.

Then $a_{1}, \ldots, a_{j}$ is a basis of the space of algebraic restrictions of closed 2 -forms to $f$.

Then we need to determine which algebraic restrictions of closed 2-forms are realizable by symplectic forms. This is possible due to the following fact. 
Proposition 3.10. Let $r$ be the minimal dimension of germs of smooth submanifolds of $\left(\mathbb{K}^{2 n}, 0\right)$ containing $\operatorname{Im} f$. Let $(S, 0)$ be one of such germs of $r$-dimensional smooth submanifolds. Let $\theta$ be the germ of a closed 2-form on $\left(\mathbb{K}^{2 n}, 0\right)$. There exists a germ of a symplectic form $\omega$ on $\left(\mathbb{K}^{2 n}, 0\right)$ such that $[\theta]_{f}=[\omega]_{f}$ if and only if $\operatorname{rank}\left(\left.\theta\right|_{T_{0} S}\right) \geq 2 r-2 n$.

4. Discrete symplectic invariants. Some new discrete symplectic invariants can be effectively calculated using algebraic restrictions. The first one is symplectic multiplicity [DJZ2] introduced in [IJ1] as a symplectic defect of a curve $f$.

Definition 4.1. The symplectic multiplicity $\mu_{\text {sympl }}(f)$ of a curve $f$ is the codimension of the symplectic orbit of $f$ in the $\mathcal{A}$-orbit of $f$.

The second one is the index of isotropy [DJZ2].

Definition 4.2. The index of isotropy $\iota(f)$ of $f$ is the maximal order of vanishing of the 2 -forms $\left.\omega\right|_{T M}$ over all smooth submanifolds $M$ contain$\operatorname{ing} \operatorname{Im} f$.

They can be described in terms of algebraic restrictions [DJZ2].

Proposition 4.3. The symplectic multiplicity of a quasi-homogeneous curve $f$ in a symplectic space is equal to the codimension of the orbit of the algebraic restriction $[\omega]_{f}$ with respect to the group of local diffeomorphisms preserving $f$ in the space of algebraic restrictions of closed 2-forms to $f$.

Proposition 4.4. The index of isotropy of a quasi-homogeneous curve $f$ in a symplectic space $\left(\mathbb{K}^{2 n}, \omega\right)$ is equal to the maximal order of vanishing of closed 2-forms representing the algebraic restriction $[\omega]_{f}$.

The above invariants are defined for the image of $f$. They have a natural generalization to any subset of the symplectic space [DJZ2].

There is one more discrete symplectic invariant introduced in [A1] which is defined specifically for a parameterized curve. This is the maximal tangency order of a curve $f$ to a smooth Lagrangian submanifold. If $H_{1}=$ $\cdots=H_{n}=0$ define a smooth submanifold $L$ in the symplectic space then the tangency order of a curve $f: \mathbb{K} \rightarrow M$ to $L$ is the minimum of the orders of vanishing at 0 of the functions $H_{1} \circ f, \ldots, H_{n} \circ f$. We denote the tangency order of $f$ to $L$ by $t(f, L)$.

Definition 4.5. The Lagrangian tangency order $\operatorname{Lt}(f)$ of a curve $f$ is the maximum of $t(f, L)$ over all smooth Lagrangian submanifolds $L$ of the symplectic space.

For a quasi-homogeneous curve $f$ with semigroup $\left(\lambda_{1}, \ldots, \lambda_{k}\right)$ the Lagrangian tangency order is greater than $\lambda_{1}$. 
$\operatorname{Lt}(f)$ is related to the index of isotropy. If the index of isotropy of $f$ is 0 then there does not exist a closed 2 -form vanishing at 0 representing the algebraic restriction of $\omega$. Then it is easy to see that the order of tangency of $f$ to $L$ is not greater than $\lambda_{k}$.

The Lagrangian tangency order of a quasi-homogeneous curve in a symplectic space can also be expressed in terms of algebraic restrictions.

The order of vanishing of the germ of a 1 -form $\alpha$ on a curve-germ $f$ at 0 is the minimum of the orders of vanishing of the functions $\alpha(X) \circ f$ at 0 over all germs of smooth vector fields $X$. If $\alpha=\sum_{i=1}^{m} g_{i} d x_{i}$ in local coordinates $\left(x_{1}, \ldots, x_{m}\right)$ then the order of vanishing of $\alpha$ on $f$ is the minimum of the orders of vanishing of the functions $g_{i} \circ f$ for $i=1, \ldots, m$.

Proposition 4.6. Let $f$ be the germ of a quasi-homogeneous curve such that the algebraic restriction of a symplectic form to it can be represented by a closed 2-form vanishing at 0 . Then the Lagrangian tangency order of the germ of a quasi-homogeneous curve $f$ is the maximum of the orders of vanishing on $f$ over all 1 -forms $\alpha$ such that $[\omega]_{f}=[d \alpha]_{f}$.

Proof. Let $L$ be the germ of a smooth Lagrangian submanifold in the standard symplectic space $\left(\mathbb{K}^{2 n}, \omega_{0}=\sum_{i=1}^{n} d p_{i} \wedge d q_{i}\right)$. Then there exist disjoint subsets $J, K \subset\{1, \ldots, n\}, J \cup K=\{1, \ldots, n\}$ and a smooth function $S\left(p_{J}, q_{K}\right)([\mathrm{AG}])$ such that

$$
L=\left\{q_{j}=-\frac{\partial S}{\partial p_{j}}\left(p_{J}, q_{K}\right): p_{k}=\frac{\partial S}{\partial q_{k}}\left(p_{J}, q_{K}\right): j \in J, k \in K\right\} .
$$

It is obvious that the order of tangency of $f$ to $L$ is equal to the order of vanishing of the 1 -form $\alpha=\sum_{k \in K} p_{k} d q_{k}-\sum_{j \in J} q_{j} d p_{j}-d S\left(p_{J}, q_{k}\right)$ and $d \alpha=\omega_{0}$.

If two closed 2-forms have the same algebraic restrictions to $f$ then their difference can be written as the differential of a 1-form vanishing on $f$ by the relative Poincaré lemma for quasi-homogeneous varieties [DJZ1]. This implies that the maximum of the orders of vanishing of 1-forms $\alpha$ on $f$ depends only on the algebraic restriction of $\omega=d \alpha$. Let $f(t)=\left(t^{\lambda_{1}}, \ldots, t^{\lambda_{k}}, 0, \ldots, 0\right)$. We may assume that $[\omega]_{f}$ may be identified with $[d \alpha]_{f}$, where $\alpha$ is a 1form on $\left\{x_{k+1}=\cdots=x_{2 n}=0\right\}$ and $\left.d \alpha\right|_{0}=0$. In local coordinates $\alpha=\sum_{i=1}^{k} g_{i} d x_{i}$ where $g_{i}$ are smooth function-germs. Let $\sigma$ be the germ of a symplectic form

$$
\sigma=d \alpha+\sum_{i=1}^{k} d x_{i} \wedge d x_{k+i}+\sum_{i=1}^{n-k} d x_{2 k+i} \wedge d x_{n+k+i} .
$$

Let $L$ be the germ of a smooth Lagrangian submanifold (with respect to $\sigma$ )

$$
\left\{x_{k+i}=g_{i}, i=1, \ldots, k, x_{2 k+j}=0, j=1, \ldots, n-k\right\} .
$$

The tangency order of $f$ to $L$ is the same as the order of vanishing of $\alpha$ on $f$. 
It is obvious that the pullback of $\sigma$ to $\left\{x_{k+1}=\cdots=x_{2 n}=0\right\}$ is $d \alpha$. Then by the Darboux-Givental theorem [AG] there exists a local diffeomorphism which is the identity on $\left\{x_{k+1}=\cdots=x_{2 n}=0\right\}$ and maps $\sigma$ to $\omega$. The submanifold $L$ is mapped to a smooth Lagrangian submanifold (with respect to the symplectic form $\omega$ ) with the same tangency order to $f$.

5. The proof of Theorem 2. In this section we prove Theorem 2. The proof is based on the following lemmas.

LEMMA 5.1. Let $N$ be the germ of a subset of $\mathbb{K}^{m}$ at 0 . Let $\left(x_{1}, \ldots, x_{m}\right)$ be a local coordinate system on $\mathbb{K}^{m}$. The space of algebraic restrictions of 2 -forms to $N$ is finite-dimensional if and only if there exists a non-negative integer $L$ such that $x_{i}^{L} d x_{j} \wedge d x_{k}$ has zero algebraic restriction to $N$ for any $i, j, k=1, \ldots, m$.

Proof. To prove the "only if" part notice that there exists a non-negative integer $K$ such that the algebraic restrictions

$$
\left[d x_{j} \wedge d x_{k}\right]_{N},\left[x_{i} d x_{j} \wedge d x_{k}\right]_{N},\left[x_{i}^{2} d x_{j} \wedge d x_{k}\right]_{N}, \ldots, \quad\left[x_{i}^{K} d x_{j} \wedge d x_{k}\right]_{N}
$$

are linearly dependent, since the space of algebraic restrictions of 2-forms to $N$ is finite-dimensional. Therefore there exist a non-negative integer $M$ and $c_{1}, \ldots, c_{s} \in \mathbb{K}$ such that $\left[x_{i}^{M}\left(1+\sum_{l=1}^{s} c_{s} x_{i}^{l}\right) d x_{j} \wedge d x_{k}\right]_{N}=0$. This implies that $\left[x_{i}^{M} d x_{j} \wedge d x_{k}\right]_{N}=0$. Now it is easy to see that $L$ is the maximum of $M$ for all choices of $i, j, k$.

To prove the "if" part first notice that any germ of a 2-form can be written in the local coordinates as $\sum_{1 \leq j<k \leq m} F_{j, k}(x) d x_{j} \wedge d x_{k}$, where $F_{j, k}(x)$ are function-germs on $\mathbb{K}^{m}$. Using Taylor expansions of $F_{j, k}(x)$ with remainder of degree greater than $m L$ we obtain the result, since $x_{1}^{i_{1}} \cdots x_{m}^{i_{m}} d x_{j} \wedge d x_{k}$ has zero algebraic restriction to $N$ for $i_{1}+\cdots+i_{m} \geq m L$.

Lemma 5.2. Let $f:(\mathbb{K}, 0) \rightarrow\left(\mathbb{K}^{2}, 0\right)$ be the germ of a $\mathbb{K}$-analytic parameterized curve in $\mathbb{K}^{2}$. Let $(y, z)$ be a local coordinate system on $\mathbb{K}^{2}$ such that the line $\{y=0\}$ does not contain $f(\mathbb{K})$. Then there exists a $\mathbb{K}$-analytic function-germ $H$ vanishing on $f$ of the form $H(y, z)=z^{p}-G(y, z) y^{l}$, where $G$ is a $\mathbb{K}$-analytic function-germ on $\mathbb{K}^{2}$, and $p, l$ are positive integers.

Proof. We use the method of construction of $H$ described in [W] (proof of Lemma 2.3.1 on page 28). As $f$ is $\mathbb{K}$-analytic, there exists a coordinate system $t$ on $\mathbb{K}$ such that $f(t)=\left(t^{m}, \sum_{i=k}^{\infty} a_{i} t^{i}\right)$. We write it as $y=t^{m}, z=$ $\sum_{i=k}^{\infty} a_{i} t^{i}$. Any non-negative integer $i$ can be written as $i=q m+r$, where $r, q$ are integers such that $0 \leq r \leq m-1$ and $q \geq 0$. Thus $z=\sum_{r=0}^{m-1} t^{r} \phi_{r}(y)$, where $\phi_{r}(y)=\sum_{q=0}^{\infty} a_{q m+r} y^{q}$ is $\mathbb{K}$-analytic for $r=0,1, \ldots, m-1$. Then we 
regard the equations

$$
t^{a} z=\sum_{r=0}^{m-a-1} t^{a+r} \phi_{r}(y)+\sum_{r=m-a}^{m-1} t^{a+r-m} z \phi_{r}(y), \quad a=0,1, \ldots, m-1,
$$

as a system of linear equations for the unknowns $t^{r}, r=0, \ldots, m-1$, with coefficients in $\mathbb{K}\{y, z\}$. The determinant $D(x, y)$ of this system has the form

$$
\operatorname{det}\left[\begin{array}{ccccc}
z-\phi_{0}(y) & -\phi_{1}(y) & -\phi_{2}(y) & \cdots & -\phi_{m-1}(y) \\
-z \phi_{m-1}(y) & z-\phi_{0}(y) & -\phi_{1}(y) & \cdots & -\phi_{m-2}(y) \\
-z \phi_{m-2}(y) & -z \phi_{m-1}(y) & z-\phi_{0}(y) & \cdots & -\phi_{m-3}(y) \\
\vdots & \vdots & \vdots & \ddots & \vdots \\
-z \phi_{1}(y) & -z \phi_{2}(y) & -z \phi_{3}(y) & \cdots & z-\phi_{0}(y)
\end{array}\right]
$$

where $\psi_{1}, \ldots, \psi_{m}$ are $\mathbb{K}$-analytic function-germs. Since the values $t^{r}$ for $r=$ $0, \ldots, m-1$ provide non-zero solutions of $(5.1)$, the determinant $D(y, z)$ vanishes on the image of the curve $f$. Since $f(0)=0$ we have $\psi_{m}(0)=0$.

Thus we can decompose $D(y, z)$ as

$$
D(y, z)=z^{m}+\psi_{1}(0) z^{m-1}+\cdots+\psi_{m-1}(0) z+y^{l} F(y, z)=z^{p} h(z)+y^{l} F(y, z),
$$

where $h$ is a polynomial of degree $m-k$ that does not vanish at $0, p, l$ are positive integers and $F$ is a $\mathbb{K}$-analytic function-germ. Now we take $H(y, z)=D(y, z) / h(z)$.

LEMMA 5.3. Let $C$ be the germ of a $\mathbb{K}$-analytic curve on $\mathbb{K}^{2}$ at 0 . Let $(y, z)$ be a local coordinate system on $\mathbb{K}^{2}$ such that the line $\{y=0\}$ does not contain any branch of $C$. Then there exists a $\mathbb{K}$-analytic function-germ $H$ vanishing on $f$ of the form $H(y, z)=z^{p}-G(y, z) y^{l}$, where $G$ is a $\mathbb{K}$-analytic function-germ on $\mathbb{K}^{2}$ and $p, l$ are positive integers.

Proof. We decompose $C$ into branches $C_{1}, \ldots, C_{s}$. Then we apply Lemma 5.2 to each branch $C_{i}$. We obtain a $\mathbb{K}$-analytic function-germ vanishing on $C_{i}$ of the form $H_{i}(y, z)=z^{p_{i}}-G_{i}(y, z) y^{l_{i}}$, where $p_{i}, l_{i}$ are positive integers and $G_{i}$ is a $\mathbb{K}$-analytic function-germ for $i=1, \ldots, s$. Now we may take $H=H_{1} \cdots H_{s}$, which vanishes on $C$ and has the desired form.

LEMMA 5.4. Let $N$ be the germ of a subset of $\mathbb{K}^{2}$ at 0 . Let $H$ be a $\mathbb{K}$-analytic function-germ on $\mathbb{K}^{2}$ vanishing on $N$. If $H$ has a regular point at 0 or an isolated critical point at 0 then the space of algebraic restrictions of 2-forms to $N$ is finite-dimensional.

Proof. The space of algebraic restrictions of 2-forms to $\{H=0\}$ is isomorphic to $\mathcal{C}_{2} /\langle H, \nabla H\rangle$ [DJZ2], where $\mathcal{C}_{2}$ is the space of $\mathbb{K}$-analytic functiongerms on $\mathbb{K}^{2}$. Thus its dimension is finite and equal to the Tjurina number 
of $\{H=0\}$. Since $N$ is a subset of $\{H=0\}$, the dimension of the space of algebraic restrictions of 2 -forms to $N$ is smaller than the Tjurina number of $\{H=0\}$, and consequently it is finite.

Proof of Theorem 2. Let $C$ be the germ of a $\mathbb{K}$-analytic curve in $\mathbb{K}^{m}$ at 0 . In fact, we prove that the vector space of algebraic restrictions of all 2 -forms to $C$ is finite-dimensional. It is obvious that the set of algebraic restrictions of closed 2-forms is a vector subspace of the vector space of algebraic restrictions of all 2-forms.

Let $\left(x_{1}, \ldots, x_{m}\right)$ be a coordinate system on $\mathbb{K}^{m}$ and let

$$
\pi_{j, k}: \mathbb{K}^{m} \ni\left(x_{1}, \ldots, x_{m}\right) \mapsto\left(x_{j}, x_{k}\right) \in \mathbb{K}^{2}
$$

be the standard projection. We choose a coordinate system so that for any $j \neq k, \pi_{j, k}(C)$ is the germ of a $\mathbb{K}$-analytic curve on $\mathbb{K}^{2}$ at 0 such that the lines $\left\{x_{j}=0\right\}$ and $\left\{x_{k}=0\right\}$ do not contain any branch of $\pi_{j, k}(C)$.

Then the space of algebraic restrictions of 2 -forms to $\pi_{j, k}(C)$ is finitedimensional by Lemma 5.4, since $\pi_{j, k}(C)$ may have a non-singular point at 0 or an isolated singular point at 0 . By Lemma 5.1 there exists a positive integer $K$ such that $x_{j}^{K} d x_{j} \wedge d x_{k}$ has zero algebraic restriction to $\pi_{j, k}(C)$ and consequently it has zero algebraic restriction to $C$.

By Lemma 5.3 there exist positive integers $p, l$ and a $\mathbb{K}$-analytic functiongerm $G$ on $\mathbb{K}^{2}$ such that the function-germ $H\left(x_{j}, x_{i}\right)=x_{i}^{p}+G\left(x_{j}, x_{i}\right) x_{j}^{l}$ vanishes on $\pi_{j, i}(C)$ and consequently it vanishes on $C$. This implies that

$$
x_{i}^{p K} d x_{j} \wedge d x_{k}=\left(-G\left(x_{j}, x_{i}\right)\right)^{K} x_{j}^{l K} d x_{j} \wedge d x_{k}
$$

also has zero algebraic restriction to $C$.

Hence by Lemma 5.1 the space of algebraic restrictions of 2 -forms to $C$ is finite-dimensional.

6. Quasi-homogeneous algebraic restrictions. In this section we prove that the action by diffeomorphisms preserving the curve is totally determined by the infinitesimal action by liftable vector fields, and the space of vector fields which act non-trivially on algebraic restrictions is a finite-dimensional vector space spanned by quasi-homogeneous liftable vector fields of bounded quasi-degrees.

The proof of Theorem 2 is very easy in the case of quasi-homogeneous parameterized curves. Let $f$ be the germ of a quasi-homogeneous curve. Then $f$ is $\mathcal{A}$-equivalent to $f(t)=\left(t^{\lambda_{1}}, \ldots, t^{\lambda_{k}}, 0, \ldots, 0\right)$. By Proposition 3.6 we consider forms in $x_{1}, \ldots, x_{k}$ coordinates only. We may also assume that the greatest common divisor $\operatorname{gcd}\left(\lambda_{1}, \ldots, \lambda_{k}\right)$ is 1 . If it is not 1 we introduce the weights $\lambda_{i} / \operatorname{gcd}\left(\lambda_{1}, \ldots, \lambda_{k}\right)$ for $x_{i}, i=1, \ldots, k$. The proof of Theorem 2 in this special case is based on the following easy observation.

Lemma 6.1. The function-germ $h(x)=x_{i}^{\lambda_{j}}-x_{j}^{\lambda_{i}}$ vanishes on $f$. 
The above lemma implies the following facts.

LEMMA 6.2. The 2 -form $x_{i}^{\lambda_{j}-1} d x_{i} \wedge d x_{j}$ has zero algebraic restriction to $f$.

Proof. By Lemma 6.1, $d h$ has zero algebraic restriction to $f$. This implies that $\left(1 / \lambda_{j}\right) d h \wedge d x_{j}=x_{i}^{\lambda_{j}-1} d x_{i} \wedge d x_{j}$ has zero algebraic restriction to $f$.

Lemma 6.3. If the monomials $s(x)=\prod_{l=1}^{k} x_{l}^{s_{l}}$ and $p(x)=\prod_{l=1}^{k} x_{l}^{p_{l}}$ have the same quasi-degree then the forms $s(x) d x_{i} \wedge d x_{j}$ and $p(x) d x_{i} \wedge d x_{j}$ have the same algebraic restrictions to $f$.

Proof. The function-germ $s(x)-p(x)$ vanishes on $f$.

The above lemmas imply that we can choose quasi-homogeneous bases of the space of algebraic restrictions of 2 -forms to $f$. Thus as a corollary of Theorem 2 and the above lemmas we obtain the following theorem.

TheOREM 6.4. The space of algebraic restrictions of closed 2-forms to the germ of a quasi-homogeneous curve $f$ is a finite-dimensional vector space spanned by algebraic restrictions of quasi-homogeneous closed 2-forms of bounded quasi-degrees.

We will use quasi-homogeneous grading on the space of algebraic restrictions. Therefore we define quasi-homogeneous algebraic restrictions.

Let $f$ be the germ of a quasi-homogeneous curve on $\left(\mathbb{K}^{m}, 0\right)$. Let $\omega$ be the germ of a $k$-form on $\left(\mathbb{K}^{m}, 0\right)$. We denote by $\omega^{(r)}$ the quasi-homogeneous part of quasi-degree $r$ in the Taylor series of $\omega$. It is easy to see that if $h$ is a function-germ on $\left(\mathbb{K}^{m}, 0\right)$ and $h \circ f=0$ then $h^{(r)} \circ f=0$ for any $r$. This simple observation implies the following proposition.

Proposition 6.5. If $[\omega]_{f}=0$ then $\left[\omega^{(r)}\right]_{f}=0$ for any $r$.

Proposition 6.5 allows us to define quasi-homogeneous algebraic restriction.

Definition 6.6. Let $a=[\omega]_{f}$ be an algebraic restriction to $f$. The algebraic restriction $a^{(r)}=\left[\omega^{(r)}\right]_{f}$ is called the quasi-homogeneous part of quasi-degree $r$ of the algebraic restriction $a$; and $a$ is quasi-homogeneous of quasi-degree $r$ if $a=a^{(r)}$.

We consider the action on the space of algebraic restrictions of closed 2 -forms by the group of diffeomorphism-germs which preserve the curve $f$ to obtain a complete symplectic classification of curves (Theorem 3.5). The tangent space to this group at the identity is given by the space of vector fields liftable over $f$. 
Definition 6.7 ([Za], [BPW]). The germ $X$ of a vector field on $\left(\mathbb{K}^{m}, 0\right)$ is called liftable over $f$ if there exists a function germ $g$ on $(\mathbb{K}, 0)$ such that

$$
g\left(\frac{d f}{d t}\right)=X \circ f
$$

The tangent space to the orbit of an algebraic restriction $a$ is given by $\mathcal{L}_{X} a$ for all vector fields $X$ liftable over $f$. The Lie derivative of an algebraic restriction with respect to a liftable vector field is well defined due to the following proposition.

Proposition 6.8. Let $X$ be the germ of a vector field on $\left(\mathbb{K}^{m}, 0\right)$ liftable over $f$ and $\omega$ be the germ of a $k$-form on $\left(\mathbb{K}^{m}, 0\right)$. If $[\omega]_{f}=0$ then $\left[\mathcal{L}_{X} \omega\right]_{f}=0$.

Proof. This is a consequence of the Cartan formula and the following fact: $d h(X) \circ f=0$ for any function-germ $h$ on $\left(\mathbb{K}^{m}, 0\right)$ vanishing on $f$. To prove the above fact notice that

$$
d h(X) \circ f=(d h \circ f)(X \circ f)=(d h \circ f) d f\left(g \frac{d}{d t}\right)=d(h \circ f)\left(g \frac{d}{d t}\right) .
$$

By the Cartan formula we also obtain the following proposition.

Proposition 6.9. If $X$ is a vector field vanishing on the image of $f$ then $\mathcal{L}_{X} a=0$ for any algebraic restriction a to $f$.

If $f$ is quasi-homogeneous then the Euler vector field $E$ is liftable over $f$. The following proposition describes its infinitesimal action on quasi-homogeneous algebraic restrictions.

Proposition 6.10. If an algebraic restriction a to $f$ is quasi-homogeneous of quasi-degree $\delta$ then $\mathcal{L}_{E} a=\delta a$.

Let $X$ be a smooth vector field. We denote by $X^{(r)}$ the quasi-homogeneous part of quasi-degree $r$ in the Taylor series of $X$. We have the following result.

Proposition 6.11. If $X$ is liftable over $f$ then $X^{(r)}$ is liftable over $f$.

Proof. We assume that $f(t)=\left(t^{\lambda_{1}}, \ldots, t^{\lambda_{k}}, 0, \ldots, 0\right)$. Then $X \circ f=$ $g(t) d f / d t$ for some function-germ $g$ on $\mathbb{K}$. This implies that

$$
X^{(r)} \circ f=\frac{1}{(r+1) !} \frac{d^{r+1} g}{d t^{r+1}}(0) t^{r+1} \frac{d f}{d t} .
$$

Let $K(f)$ be the minimal natural number such that all quasi-homogeneous algebraic restrictions to $f$ of closed 2-forms of quasi-degree greater than $K(f)$ vanish. By Theorem 6.4, $K(f)$ is finite.

Theorem 6.12. Let $f(t)=\left(t^{\lambda_{1}}, \ldots, t^{\lambda_{k}}, 0, \ldots, 0\right)$. Let $X_{s}$ be the germ of a vector field such that $X_{s} \circ f=t^{s+1} d f / d t$. Then the tangent space to the orbit of the quasi-homogeneous algebraic restriction $a_{r}$ of quasi-degree $r$ is 
spanned by $\mathcal{L}_{X_{s}} a_{r}$ for $s$ that are $\mathbb{Z}_{\geq 0}$-linear combinations of $\lambda_{1}, \ldots, \lambda_{k}$ and are smaller than $K(f)-r$.

Proof. Let $Y$ be a quasi-homogeneous vector field liftable over $f$. Then $Y \circ f=c t^{s+1} d f / d t$ where $s$ is the quasi-degree of $Y$ and $c \in \mathbb{K}$. By Proposition 6.9 we obtain $\mathcal{L}_{Y} a_{r}=c \mathcal{L}_{X_{s}} a_{r}$, since $\left(Y-c X_{s}\right) \circ f=0$. If $Z$ is a liftable vector field we can decompose it to $\sum_{s=0}^{K(f)-r-1} Z^{(s)}+V$, where $V$ is a liftable vector field such that $V^{(s)}=0$ for $s<K(f)-r$. Then $\mathcal{L}_{Z} a_{r}=\sum_{s=0}^{K(f)-r-1} c_{s} \mathcal{L}_{X_{s}} a_{r}+\mathcal{L}_{V} a_{r}$, where $c_{s} \in \mathbb{K}$ for $s=0, \ldots, K(f)-r-1$. Proposition 2.5 implies that $\left(\mathcal{L}_{V} a_{r}\right)^{(s)}=0$ for $s<K(f)$. By Taylor expansion we can decompose $\mathcal{L}_{V} a_{r}$ as $\sum_{i=1}^{m} f_{i} b_{i}$, where $f_{i}$ are function-germs and $b_{i}$ are quasi-homogeneous algebraic restrictions of quasi-degree greater than $K(f)-1$. Thus $\mathcal{L}_{V} a_{r}=0$.

Theorem 6.12 implies Theorem 3. Now we prove the following theorem which is crucial for the description of the action of diffeomorphisms preserving $f$ on the space of algebraic restrictions to $f$.

TheOREM 6.13. Let $a_{1}, \ldots, a_{p}$ be a quasi-homogeneous basis of quasidegrees $\delta_{1} \leq \cdots \leq \delta_{s}<\delta_{s+1} \leq \cdots \leq \delta_{p}$ of the space of algebraic restrictions of closed 2-forms to $f$. Let $a=\sum_{j=s}^{p} c_{j} a_{j}$, where $c_{j} \in \mathbb{K}$ for $j=s, \ldots, p$ and $c_{s} \neq 0$. If there exists a liftable quasi-homogeneous vector field $X$ over $f$ such that $\mathcal{L}_{X} a_{s}=r a_{k}$ for $k>s$ and $r \neq 0$ then $a$ is diffeomorphic to $\sum_{j=s}^{k-1} c_{j} a_{j}+\sum_{j=k+1}^{p} b_{j} a_{j}$ for some $b_{j} \in \mathbb{K}, j=k+1, \ldots, p$.

Proof. We present the proof for $\mathbb{K}=\mathbb{R}$. In the $\mathbb{C}$-analytic category the proof is similar. We use the Moser homotopy method. Let $a_{t}=\sum_{j=s}^{k-1} c_{j} a_{j}+$ $(1-t) c_{k} a_{k}+\sum_{j=k+1}^{p} b_{j}(t) a_{j}$ where $b_{j}(t)$ are smooth (or $\mathbb{R}$-analytic) functions $b_{j}:[0 ; 1] \rightarrow \mathbb{K}$ such that $b_{j}(0)=c_{j}$ for $j=k+1, \ldots, p$. Let $\Phi_{t}, t \in[0 ; 1]$, be the flow of the vector field $\frac{c_{k}}{r c_{s}} V$. We show that there exist functions $b_{j}$ such that

$$
\Phi_{t}^{*} a_{t}=a
$$

for $t \in[0 ; 1]$. Differentiating (6.1) we obtain

$$
\mathcal{L}_{\frac{c_{k}}{r c_{s}} X} a_{t}=c_{k} a_{k}-\sum_{j=k+1}^{p} \frac{d b_{j}}{d t} a_{j} .
$$

Since $\mathcal{L}_{X} a_{s}=r a_{k}$, the quasi-degree of $X$ is $\delta_{k}-\delta_{s}$. Hence the quasi-degree of $\mathcal{L}_{\frac{c_{k}}{r c_{s}} X} a_{j}$ is greater than $\delta_{k}$ for $j>s$. Then the $b_{j}$ are solutions of the system of $p-k$ first order linear ODEs defined by (6.2) with the initial data $b_{j}(0)=c_{j}$ for $j=k+1, \ldots, p$. This implies that $a_{0}=a$ and $a_{1}=$ $\sum_{j=s}^{k-1} c_{j} a_{j}+\sum_{j=k+1}^{p} b_{j}(1) a_{j}$ are diffeomorphic. 
Remarks on the algorithm for a quasi-homogeneous parameterized curve with an arbitrary semigroup. The results of this section allow us to give an algorithm for the classification of the symplectic singularities of an arbitrary quasi-homogeneous parameterized curve-germ $f$. It is obvious that this algorithm depends only on the semigroup of the curve singularity.

Let us assume that the semigroup is

$$
\left(\lambda_{1}, \ldots, \lambda_{k}\right),
$$

where $\lambda_{1}<\cdots<\lambda_{k}$ are positive integers linearly independent over nonnegative integers. We use the quasi-homogeneous grading on the space of algebraic restrictions of 2 -forms with weights $\left(\lambda_{1}, \ldots, \lambda_{k}\right)$ for coordinates $\left(x_{1}, \ldots, x_{k}\right)$. We may also assume that $\lambda_{1}, \ldots, \lambda_{k}$ are relatively prime. If they are not we introduce weights $\lambda_{i} / \operatorname{gcd}\left(\lambda_{1}, \ldots, \lambda_{k}\right)$ for $x_{i}, i=1, \ldots, k$.

We fix the quasi-degree $\delta$ starting with $\lambda_{1}+\lambda_{2}$ since there are no quasihomogeneous 2 -forms with a smaller quasi-degree.

2 -forms of quasi-degree $\delta$ (together with the zero 2-form) form a finitedimensional subspace of the space of differential 2-forms.

By Lemma 6.3 the algebraic restrictions of all forms of quasi-degree $\delta$ of the form

$$
s(x) d x_{i} \wedge d x_{j}
$$

for fixed $i \neq j$ are linearly dependent. Hence for all $i<j$ we need to check whether the equation

$$
a_{1} \lambda_{1}+\cdots+a_{k} \lambda_{k}=\delta-\lambda_{i}-\lambda_{j}
$$

has a solution $a_{1}, \ldots, a_{k}$ in non-negative integers.

For fixed $i<j$ we take one of the solutions of (6.4) (if it exists). All other algebraic restrictions of the 2 -forms of the form (6.3) are linear combinations of $\left[x_{1}^{a_{1}} \cdots x_{k}^{a_{k}} d x_{i} \wedge d x_{j}\right]_{f}$.

To find a basis of algebraic restrictions of quasi-homogeneous 2-forms with quasi-degree $\delta$ we look for quasi-homogeneous functions vanishing on $f$.

To find them we need to solve the equation

$$
a_{1} \lambda_{1}+\cdots+a_{k} \lambda_{k}=\delta-\lambda_{i} .
$$

If $\left(a_{1}, \ldots, a_{k}\right)$ and $\left(b_{1}, \ldots, b_{k}\right)$ are distinct solution of (6.5) then the functiongerm

$$
H\left(x_{1}, \ldots, x_{k}\right)=x_{1}^{a_{1}} \cdots x_{k}^{a_{k}}-x_{1}^{b_{1}} \cdots x_{k}^{b_{k}}
$$

vanishes on $f$ and the form $d H \wedge d x_{i}$ has zero algebraic restriction to $f$ and has quasi-degree $\delta$. If we also consider quasi-homogeneous 2 -forms of quasi-degree $\delta-s$ multiplied by monomials of quasi-degree $s$ then we obtain all relations between algebraic restrictions of quasi-homogeneous 2-forms of quasi-degree $\delta$ and consequently we find a basis of this vector space. 
Then we proceed to algebraic restrictions with quasi-degree $\delta+1$.

For some quasi-degrees we find that all quasi-homogeneous 2-forms have zero algebraic restriction to $f$. Then using the fact that quasi-homogeneous forms of a sufficiently high quasi-degree can be obtained by multiplication by functions of quasi-homogeneous forms of lower degrees we infer that all 2 -forms of a sufficiently high quasi-degree have zero algebraic restriction. In this way we construct a quasi-homogeneous basis of the space of algebraic restrictions of all 2-forms.

Then by Proposition 3.9 we get a quasi-homogeneous basis of the space of algebraic restrictions of closed 2-forms from the quasi-homogeneous basis of the space of algebraic restrictions of all 2 -forms.

Next we calculate the number $K(f)$ and we find germs of vector fields such that $X_{s} \circ f=t^{s+1} d f / d t$ for $s$ representable as non-negative integer combinations of $\lambda_{1}, \ldots, \lambda_{k}$ and smaller than $K(f)-\lambda_{1}-\lambda_{2}$. By Theorem 6.12 the tangent space to the orbit of the quasi-homogeneous algebraic restriction $a_{r}$ of quasi-degree $r$ is spanned by $\mathcal{L}_{X_{s}} a_{r}$.

Finally, we apply Theorem 6.13 to get a classification of the algebraic restrictions. From this classification we easily obtain the symplectic singularities normal forms.

In the next sections we apply the above algorithm to curves with semigroups $(3,4,5),(3,5,7)$ and $(3,7,8)$.

Although the algorithm works very well for concrete examples, the problem of calculating the dimension of the space of algebraic restrictions of closed 2-forms to a quasi-homogeneous parameterized curve in terms of the semigroup of this curve is complicated. It is obvious that it is related to the classical Frobenius coin problem (the diophantine Frobenius problem) $[R]$.

Frobenius Coin Problem. Given $k$ relatively prime positive integers $\lambda_{1}, \ldots, \lambda_{k}$, find the largest natural number (denoted by $g\left(\lambda_{1}, \ldots, \lambda_{k}\right)$ and called the Frobenius number) that is not representable as a non-negative integer combination of $\lambda_{1}, \ldots, \lambda_{k}$.

By Schur's theorem the Frobenius number is finite $[\mathrm{R}]$. The formula for the Frobenius number for $k=2$ was found by J. J. Sylvester: $g\left(\lambda_{1}, \lambda_{2}\right)=$ $\lambda_{1} \lambda_{2}-\lambda_{1}-\lambda_{2}([\mathrm{~S}])$. He also demonstrated that there are $\left(\lambda_{1}-1\right)\left(\lambda_{2}-1\right) / 2$ non-representable natural numbers. More complicated formulas and fast algorithms to calculate the Frobenius number for $k=3$ are known, but the general problem for arbitrary $k$ is known to be NP-hard [R].

7. Symplectic singularities of curves with semigroup $(3,4,5)$. In this section we apply the results of the previous section to prove the following theorem. 
THEOREM 7.1. Let $\left(\mathbb{R}^{2 n}, \omega_{0}=\sum_{i=1}^{n} d p_{i} \wedge d q_{i}\right)$ be the symplectic space with the canonical coordinates $\left(p_{1}, q_{1}, \ldots, p_{n}, q_{n}\right)$. Then the germ of a curve $f:(\mathbb{R}, 0) \rightarrow\left(\mathbb{R}^{2 n}, 0\right)$ with semigroup $(3,4,5)$ is symplectically equivalent to one and only one of the curves in the second column of Table 1 for $n>2$, and $f$ is symplectically equivalent to one and only one of the curves in the second column and rows 1-3 for $n=2$.

The symplectic multiplicity, the index of isotropy and the Lagrangian tangency order are given in the third, fourth and fifth columns of Table 1.

Table 1. Symplectic classification of curves with semigroup $(3,4,5)$

\begin{tabular}{llccc}
\hline & Normal form of $f$ & $\mu_{\text {sympl }}(f)$ & $\iota(f)$ & $\operatorname{Lt}(f)$ \\
\hline 1 & $t \mapsto\left(t^{3}, t^{4}, t^{5}, 0, \ldots, 0\right)$ & 0 & 0 & 4 \\
2 & $t \mapsto\left(t^{3}, \pm t^{5}, t^{4}, 0, \ldots, 0\right)$ & 1 & 0 & 5 \\
3 & $t \mapsto\left(t^{3}, 0, t^{4}, t^{5}, 0, \ldots, 0\right)$ & 2 & 0 & 5 \\
4 & $t \mapsto\left(t^{3}, \pm t^{7}, t^{4}, 0, t^{5}, 0, \ldots, 0\right)$ & 3 & 1 & 7 \\
5 & $t \mapsto\left(t^{3}, t^{8}, t^{4}, 0, t^{5}, 0, \ldots, 0\right)$ & 4 & 1 & 8 \\
6 & $t \mapsto\left(t^{3}, 0, t^{4}, 0, t^{5}, 0, \ldots, 0\right)$ & 5 & $\infty$ & $\infty$ \\
\hline
\end{tabular}

We use the method of algebraic restrictions. The germ of a curve $f$ : $\mathbb{R} \ni t \mapsto f(t) \in \mathbb{R}^{2 n}$ with semigroup $(3,4,5)$ is $\mathcal{A}$-equivalent to $t \mapsto$ $\left(t^{3}, t^{4}, t^{5}, 0, \ldots, 0\right)$. First we calculate the space of algebraic restrictions of 2 -forms to the image of $f$ in $\mathbb{R}^{2 n}$.

Proposition 7.2. The space of algebraic restrictions of differential 2forms to $f$ is the 6-dimensional vector space spanned by the following algebraic restrictions:

$$
\begin{array}{rlrl}
a_{7} & =\left[d x_{1} \wedge d x_{2}\right]_{g}, \\
a_{10} & =\left[x_{1} d x_{1} \wedge d x_{2}\right]_{g}, & a_{8} & =\left[d x_{3} \wedge d x_{1}\right]_{g}, \\
a_{11} & =\left[x_{2} d x_{1} \wedge d x_{2}\right]_{g}, & a_{9} & =\left[d x_{2} \wedge d x_{3}\right]_{g}, \\
a_{12} & =\left[x_{1} d x_{2} \wedge d x_{3}\right]_{g},
\end{array}
$$

where $\delta$ is the quasi-degree of $a_{\delta}$.

Proof. The image of $f$ is contained in the 3-dimensional smooth submanifold $\left\{x_{\geq 4}=0\right\}$. By Proposition 3.6 we can restrict our considerations to $\mathbb{R}^{3}$ and the curve $g: \mathbb{R} \ni t \mapsto\left(t^{3}, t^{4}, t^{5}\right) \in \mathbb{R}^{3}$, which is quasihomogeneous with weights $3,4,5$ for variables $x_{1}, x_{2}, x_{3}$. We use the quasihomogeneous grading on the space of algebraic restrictions of differential 2 -forms to $g(t)=\left(t^{3}, t^{4}, t^{5}\right)$ with these weights. It is easy to see that the quasi-homogeneous functions or 2-forms of a fixed quasi-degree form a finitedimensional vector space. The same is true for quasi-homogeneous algebraic restrictions of 2 -forms of a fixed quasi-degree.

There are no quasi-homogeneous function-germs on $\mathbb{R}^{3}$ vanishing on $g$ of quasi-degree less than 8 . The vector space of quasi-homogeneous function- 
Table 2. Quasi-homogeneous function-germs of quasidegree 8, 9, 10 vanishing on the curve $t \mapsto\left(t^{3}, t^{4}, t^{5}\right)$

\begin{tabular}{ccc}
\hline Quasi-degree $\delta$ & $f_{\delta}$ & Differential $d f_{\delta}$ \\
\hline 8 & $x_{1} x_{3}-x_{2}^{2}$ & $x_{1} d x_{3}+x_{3} d x_{1}-2 x_{2} d x_{2}$ \\
9 & $x_{2} x_{3}-x_{1}^{3}$ & $x_{2} d x_{3}+x_{3} d x_{2}-3 x_{1}^{2} d x_{1}$ \\
10 & $x_{1}^{2} x_{2}-x_{3}^{2}$ & $x_{1}^{2} d x_{2}+2 x_{1} x_{2} d x_{1}-2 x_{3} d x_{3}$ \\
\hline
\end{tabular}

Table 3. The quasi-homogeneous basis of algebraic restrictions of 2-forms to the curve $t \mapsto\left(t^{3}, t^{4}, t^{5}\right)$

\begin{tabular}{|c|c|c|c|c|}
\hline$\delta$ & Basis & Forms & Relations & Proof \\
\hline 7 & $a_{7}$ & $\alpha_{7}=d x_{1} \wedge d x_{2}$ & $a_{7}:=\left[\alpha_{7}\right]_{g}$ & \\
\hline 8 & $a_{8}$ & $\alpha_{8}=d x_{3} \wedge d x_{1}$ & $a_{8}:=\left[\alpha_{8}\right]_{g}$ & \\
\hline 9 & $a_{9}$ & $\alpha_{9}=d x_{2} \wedge d x_{3}$ & $a_{9}:=\left[\alpha_{9}\right]_{g}$ & \\
\hline 10 & $a_{10}$ & $x_{1} \alpha_{7}$ & $a_{10}:=x_{1} a_{7}$ & \\
\hline 11 & $a_{11}$ & $\begin{array}{l}x_{2} \alpha_{7} \\
x_{1} \alpha_{8}\end{array}$ & $\begin{array}{c}a_{11}:=x_{2} a_{7} \\
a_{11}=-2 x_{1} a_{8}\end{array}$ & {$\left[d f_{8} \wedge d x_{1}\right]_{g}=0$} \\
\hline 12 & $a_{12}$ & $\begin{array}{l}x_{3} \alpha_{7} \\
x_{2} \alpha_{8} \\
x_{1} \alpha_{9} \\
\end{array}$ & $\begin{aligned} a_{12} & :=x_{3} a_{7} \\
a_{12} & =x_{2} a_{8} \\
a_{12} & =x_{1} a_{9}\end{aligned}$ & $\begin{array}{l}{\left[d f_{9} \wedge d x_{1}\right]_{g}=0} \\
{\left[d f_{8} \wedge d x_{2}\right]_{g}=0}\end{array}$ \\
\hline 13 & 0 & $\begin{array}{l}x_{1}^{2} \alpha_{7} \\
x_{3} \alpha_{8} \\
x_{2} \alpha_{9} \\
\end{array}$ & $\begin{array}{l}x_{1}^{2} a_{7}=0 \\
x_{3} a_{8}=0 \\
x_{2} a_{9}=0\end{array}$ & $\begin{array}{c}{\left[d f_{10} \wedge d x_{1}\right]_{g}=0} \\
{\left[d f_{9} \wedge d x_{2}\right]_{g}=0} \\
{\left[d f_{8} \wedge d x_{3}\right]_{g}=0}\end{array}$ \\
\hline 14 & 0 & $\begin{array}{c}x_{1} x_{2} \alpha_{7} \\
x_{1}^{2} \alpha_{8} \\
x_{2} \alpha_{10}\end{array}$ & $\begin{array}{c}x_{1} x_{2} a_{7}=0 \\
x_{1}^{2} a_{8}=0 \\
x_{2} a_{10}=0\end{array}$ & $\begin{array}{c}{\left[d f_{10} \wedge d x_{2}\right]_{g}=0} \\
{\left[d f_{9} \wedge d x_{3}\right]_{g}=0} \\
x_{1}\left[d f_{8} \wedge d x_{1}\right]_{g}=0\end{array}$ \\
\hline 15 & 0 & $\begin{array}{c}x_{1} x_{3} \alpha_{7}, \\
x_{1} x_{2} \alpha_{8} \\
x_{1}^{2} \alpha_{9}\end{array}$ & $\begin{array}{c}x_{1} x_{3} a_{7}=0 \\
x_{1} x_{2} a_{8}=0 \\
x_{1}^{2} a_{9}=0\end{array}$ & $\begin{array}{c}{\left[d f_{10} \wedge d x_{3}\right]_{g}=0} \\
x_{1}\left[d f_{9} \wedge d x_{1}\right]_{g}=0 \\
x_{1}\left[d f_{8} \wedge d x_{2}\right]_{g}=0\end{array}$ \\
\hline$\geq 16$ & 0 & $\begin{array}{l}x_{1} \beta_{\geq 13}, \\
x_{2} \beta_{\geq 12} \\
x_{3} \beta_{\geq 11}\end{array}$ & $\begin{array}{c}b_{\geq 13}:=\left[\beta_{\geq 13}\right]_{g} \\
x_{1} b_{\geq 13}=0 \\
b_{\geq 12}:=\left[\beta_{\geq 12}\right]_{g} \\
x_{2} b_{\geq 12}=0 \\
b_{\geq 11}:=\left[\beta_{\geq 11}\right]_{g} \\
x_{3} b_{\geq 11}=0\end{array}$ & $\begin{array}{c}x_{2} b_{\geq 12}=x_{1} b_{\geq 13}^{\prime} \\
x_{3} b_{\geq 11}=x_{1} b_{\geq 13}^{\prime \prime} \\
\delta\left(b_{\geq 13}\right) \geq 13 \\
b_{\geq 13}=0\end{array}$ \\
\hline
\end{tabular}

germs of degree $i=8,9$, 10 vanishing on $g$ is spanned by $f_{i}$ given in Table 2 together with their differentials. We do not need to consider quasihomogeneous function-germs of higher quasi-degree, since using $f_{8}, f_{9}$ and $f_{10}$ we show that the algebraic restrictions of quasi-homogeneous 2 -forms of 
quasi-degree greater than 12 are zero (see Table 3) and all possible relations of algebraic restrictions of quasi-homogeneous 2-forms of quasi-degree less than 13 are generated by quasi-homogeneous functions vanishing on $g$ of quasi-degree less than $13-3=10$.

Now we can calculate the space of algebraic restrictions of 2 -forms. The scheme of the proof is shown in Table 3 . The first column lists the possible degrees $\delta$ of a 2 -form. In the second column there is a basis of the algebraic restrictions of 2 -forms of degree $\delta$. In the third column we give a basis of 2 -forms of degree $\delta$. In the fourth column we show the relations between algebraic restrictions of elements of the basis of 2 -forms of degree $\delta$. The last column sketches the proofs of these relations.

The lowest possible quasi-degree of a 2 -form is 7 . The space of quasihomogeneous 2 -forms of degree 7 is spanned by $d x_{1} \wedge d x_{2}$. This form does not have zero algebraic restriction since it does not vanish at 0 [DJZ2]. This implies that the vector space of algebraic restrictions of 2 -forms of quasidegree 7 is spanned by $\left[d x_{1} \wedge d x_{2}\right]_{g}$. We have a similar situation for quasidegrees $8,9,10$. The algebraic restriction $x_{1} a_{7}$ is not zero since there are no quasi-homogeneous functions vanishing on $g$ of quasi-degree not greater than $10-3=7$.

The space of quasi-homogeneous 2-forms of quasi-degree 11 is spanned by $x_{2} d x_{1} \wedge d x_{2}$ and $x_{1} d x_{3} \wedge d x_{1}$. But by Proposition 3.8 we have $\left[d f_{8} \wedge d x_{1}\right]_{9}=0$, which implies that the algebraic restrictions of these 2 -forms are linearly dependent: $x_{1} a_{8}=\left[x_{1} d x_{3} \wedge d x_{1}\right]_{g}=\left[-2 x_{2} d x_{1} \wedge d x_{2}\right]_{g}=-2 a_{11}$. We use similar arguments to show that the space of algebraic restrictions of quasidegree 12 is spanned by $a_{12}$.

The space of 2-forms of quasi-degree 13 is 3 -dimensional. But from the linearly independent linear relations satisfied by algebraic restrictions of the elements of the basis, given in the last column of the row for $\delta=13$, we see that all algebraic restrictions of quasi-degree 13 are zero. The same arguments apply for quasi-degrees 14 and 15 .

To prove that all algebraic restrictions of quasi-degree 16 are 0 we notice that they can have the following forms of quasi-degree 16: $x_{1} \beta_{13}$ or $x_{2} \beta_{12}$ or $x_{3} \beta_{11}$. In the first case the algebraic restriction $b_{13}=\left[\beta_{13}\right]_{g}$ has quasi-degree 13 , so it is 0 . In the second case the quasi-degree of $b_{12}=\left[\beta_{12}\right]_{g}$ is 12 . So the algebraic restriction $b_{12}$ can be represented in the form $c x_{1} a_{9}$, where $c \in \mathbb{R}$. But then $x_{2} b_{12}=x_{1}\left(c x_{2} a_{9}\right)$. The quasi-degree of $c x_{2} a_{9}$ is 13 , which implies that $c x_{2} a_{9}$ is 0 . A similar shows that $x_{3} b_{11}$ is 0 . Using the same arguments and induction on the quasi-degree we show that all algebraic restrictions of higher quasi-degree are 0.

Any smooth 2 -form $\omega$ can be decomposed as $\omega=\sum_{i=7}^{12} \omega_{i}+\sum_{j=1}^{k} f_{j} \sigma_{j}$, where $k$ is a positive integer, $\omega_{i}$ is a quasi-homogeneous 2-form of quasi- 
degree $i$ for $i=7, \ldots, 12$ and $f_{j}$ are smooth function-germs and $\sigma_{j}$ are quasihomogeneous 2 -forms of quasi-degree greater than 12 for $j=1, \ldots, k$. Thus the space of algebraic restrictions of 2 -forms is spanned by $a_{7}, \ldots, a_{12}$.

Proposition 7.3. The space of algebraic restrictions of closed differential 2-forms to the image of $f$ is the 5-dimensional vector space spanned by the following algebraic restrictions:

$$
a_{7}, a_{8}, a_{9}, a_{10}, a_{11}
$$

Proof. It is easy to see that $d a_{i}=0$ for $i<12$ and $d a_{12} \neq 0$. Then we apply Proposition 3.9.

Proposition 7.4. Any algebraic restriction of a symplectic form to $f$ is diffeomorphic to one and only one of $a_{7}, a_{8},-a_{8}, a_{9}, a_{10},-a_{10}, a_{11}, 0$.

Proof. By Theorem 6.12 we consider vector fields $X_{s}$ such that $X_{s} \circ f=$ $t^{s+1} d f / d t$ for $s=0, \ldots, 5$. They have the following form:

$$
\begin{gathered}
X_{0}=E=3 x_{1} \frac{\partial}{\partial x_{1}}+4 x_{2} \frac{\partial}{\partial x_{2}}+5 x_{3} \frac{\partial}{\partial x_{3}}, \quad X_{1}=3 x_{2} \frac{\partial}{\partial x_{1}}+4 x_{3} \frac{\partial}{\partial x_{2}}+5 x_{1}^{2} \frac{\partial}{\partial x_{3}}, \\
X_{2}=3 x_{3} \frac{\partial}{\partial x_{1}}+4 x_{1}^{2} \frac{\partial}{\partial x_{2}}+5 x_{1} x_{2} \frac{\partial}{\partial x_{3}}, \quad X_{3}=x_{1} E, \quad X_{4}=x_{2} E .
\end{gathered}
$$

Table 4. Infinitesimal actions on algebraic restrictions of closed 2-forms to the curve $t \mapsto\left(t^{3}, t^{4}, t^{5}\right)$

\begin{tabular}{|c|ccccc|}
\hline $\mathcal{L}_{X_{i}} a_{j}$ & $a_{7}$ & $a_{8}$ & $a_{9}$ & $a_{10}$ & $a_{11}$ \\
\hline$X_{0}=E$ & $7 a_{7}$ & $8 a_{8}$ & $9 a_{9}$ & $10 a_{10}$ & $11 a_{11}$ \\
$X_{1}$ & $-4 a_{8}$ & $-3 a_{9}$ & $-10 a_{10}$ & $11 a_{11}$ & 0 \\
$X_{2}$ & $-3 a_{9}$ & $-5 a_{10}$ & $11 a_{11}$ & 0 & 0 \\
$X_{3}=x_{1} E$ & $10 a_{10}$ & $-22 a_{11}$ & 0 & 0 & 0 \\
$X_{4}=x_{2} E$ & $11 a_{11}$ & 0 & 0 & 0 & 0 \\
\hline
\end{tabular}

The infinitesimal action of these germs of quasi-homogeneous liftable vector fields on the basis of the vector space of algebraic restrictions of closed 2 -forms to $f$ is given in Table 4 . Using the data of Table 4 we deduce by Theorem 6.13 that an algebraic restriction of the form $\sum_{i \geq s} c_{i} a_{i}$ for $c_{s} \neq 0$ is diffeomorphic to $c_{s} a_{s}$. Finally, we reduce $c_{s} a_{s}$ to $a_{s}$ if the quasi-degree $s$ is odd or to $\operatorname{sgn}\left(c_{s}\right) a_{s}$ if $s$ is even by the diffeomorphism $\Phi_{t}\left(x_{1}, x_{2}, x_{3}\right)=$ $\left(t^{3} x_{1}, t^{4} x_{2}, t^{5} x_{3}\right)$ for $t=c_{s}^{1 / s}$ or for $t=\left|c_{s}\right|^{1 / s}$ respectively.

The algebraic restrictions $a_{8},-a_{8}$ are not diffeomorphic. Indeed, any diffeomorphism $\Phi=\left(\Phi_{1}, \ldots, \Phi_{2 n}\right)$ of $\left(\mathbb{R}^{2 n}, 0\right)$ preserving $f(t)=\left(t^{3}, t^{4}, t^{5}, 0, \ldots, 0\right)$ 
has linear part

$$
\begin{array}{r}
A^{3} x_{1}+A_{12} x_{2}+A_{13} x_{3}+A_{14} x_{4}+\cdots+A_{1,2 n} x_{2 n} \\
A^{4} x_{2}+A_{23} x_{3}+A_{24} x_{4}+\cdots+A_{2,2 n} x_{2 n} \\
A^{5} x_{3}+A_{34} x_{4}+\cdots+A_{3,2 n} x_{2 n} \\
A_{44} x_{4}+\cdots+A_{4,2 n} x_{2 n} \\
\vdots \quad \vdots \quad \vdots \quad \vdots \quad \vdots \\
A_{2 n, 4} x_{4}+\cdots+A_{2 n, 2 n} x_{2 n}
\end{array}
$$

where $A, A_{i, j} \in \mathbb{R}$. If we assume that $\Phi^{*}\left(a_{8}\right)=-a_{8}$, then $\left.A^{8} d x_{3} \wedge d x_{1}\right|_{0}=$ $-\left.d x_{3} \wedge d x_{1}\right|_{0}$, which is a contradiction.

One can similarly prove that $a_{10},-a_{10}$ are not diffeomorphic.

Proof of Theorem 7.1. Let $\theta_{i}$ be a 2 -form on $\mathbb{R}^{3}$ such that $a_{i}=\left[\theta_{i}\right]_{g}$. Then $\operatorname{rank}\left(\left.\theta_{i}\right|_{0}\right) \geq 2$ if $n=2$ and $\operatorname{rank}\left(\left.\theta_{i}\right|_{0}\right) \geq 0$ if $n>2$ by Proposition 3.10. It is easy to see that $a_{7}, \pm a_{8}, a_{9}$ are realizable by the symplectic forms

$$
\begin{gathered}
d x_{1} \wedge d x_{2}+d x_{3} \wedge d x_{4}+\cdots, \quad \pm d x_{3} \wedge d x_{1}+d x_{2} \wedge d x_{4}+\cdots, \\
d x_{2} \wedge d x_{3}+d x_{1} \wedge d x_{4}+\cdots
\end{gathered}
$$

respectively. The algebraic restrictions $\pm a_{10}, a_{11}$ and $a_{\infty}=0$ are realizable by the forms

$$
\begin{gathered}
\pm x_{1} d x_{1} \wedge d x_{2}+d x_{1} \wedge d x_{4}+d x_{2} \wedge d x_{5}+d x_{3} \wedge d x_{6}+\cdots \\
x_{2} d x_{1} \wedge d x_{2}+d x_{1} \wedge d x_{4}+d x_{2} \wedge d x_{5}+d x_{3} \wedge d x_{6}+\cdots \\
d x_{1} \wedge d x_{4}+d x_{2} \wedge d x_{5}+d x_{3} \wedge d x_{6}+\cdots
\end{gathered}
$$

respectively. By a simple coordinate change we map the above forms to the Darboux normal form and we obtain the normal forms of the curve.

By Propositions 4.3, 7.3, 7.4 and using the data in Table 4 we obtain the symplectic multiplicities of curves in Table 1 . The indices of isotropy for these curves are calculated via Propositions 4.4 and 7.4. The Lagrangian tangency orders for the curves in rows $1-3$ are obtained using the fact that any Lagrangian submanifold can be represented in the form (4.1). By Propositions 4.6 and 7.4 we obtain this invariant for other curves in Table 1.

8. Symplectic singularities of curves with semigroup $(3,5,7)$. In this section we present a symplectic classification of curves with semigroup $(3,5,7)$.

TheOrem 8.1. Let $\left(\mathbb{R}^{2 n}, \omega_{0}=\sum_{i=1}^{n} d p_{i} \wedge d q_{i}\right)$ be the symplectic space with the canonical coordinates $\left(p_{1}, q_{1}, \ldots, p_{n}, q_{n}\right)$. Then the germ of a curve $f:(\mathbb{R}, 0) \rightarrow\left(\mathbb{R}^{2 n}, 0\right)$ with semigroup $(3,5,7)$ is symplectically equivalent to one and only one of the curves in the second column of Table 5 for $n>2$ 
and $f$ is symplectically equivalent to one and only one of the curves in the second column and rows $1-3$ and 5 for $n=2$. The parameter $c$ is a modulus.

The symplectic multiplicity, the index of isotropy and the Lagrangian tangency order are given in the third, fourth and fifth columns of Table 5.

Table 5. Symplectic classification of curves with semigroup $(3,5,7)$

\begin{tabular}{llccc}
\hline & Normal form of $f$ & $\mu_{\text {sympl }}(f)$ & $\iota(f)$ & $\operatorname{Lt}(f)$ \\
\hline 1 & $t \mapsto\left(t^{3}, \pm t^{5}, t^{7}, 0, \ldots, 0\right)$ & 0 & 0 & 5 \\
2 & $t \mapsto\left(t^{3}, \pm t^{7}, t^{5}, c t^{6}, \ldots, 0\right)$ & 2 & 0 & 7 \\
3 & $t \mapsto\left(t^{3}, t^{8}, t^{5}, c t^{7}, \ldots, 0\right), c \neq 0$ & 3 & 0 & 7 \\
4 & $t \mapsto\left(t^{3}, t^{8}, t^{5}, 0, t^{7}, 0, \ldots, 0\right)$ & 3 & 1 & 8 \\
5 & $t \mapsto\left(t^{3}, c t^{10}, \pm t^{5}, t^{7}, 0, \ldots, 0\right)$ & 4 & 0 & 7 \\
6 & $t \mapsto\left(t^{3}, c t^{11}, t^{5}, t^{8}, t^{7}, 0, \ldots, 0\right)$ & 5 & 1 & 10 \\
7 & $t \mapsto\left(t^{3}, \pm t^{11}, t^{5}, 0, t^{7}, 0, \ldots, 0\right)$ & 5 & 2 & 11 \\
8 & $t \mapsto\left(t^{3}, \pm t^{13} / 2, t^{5}, 0, t^{7}, 0, \ldots, 0\right)$ & 6 & 2 & 13 \\
9 & $t \mapsto\left(t^{3}, 0, t^{5}, 0, t^{7}, 0, \ldots, 0\right)$ & 7 & $\infty$ & $\infty$ \\
\hline
\end{tabular}

The germ of a curve $f: \mathbb{R} \ni t \mapsto f(t) \in \mathbb{R}^{2 n}$ with semigroup $(3,5,7)$ is $\mathcal{A}$-equivalent to $t \mapsto\left(t^{3}, t^{5}, t^{7}, 0, \ldots, 0\right)$. We use the same method as in the previous section to obtain a symplectic classification of these curves. We only present the main steps with all calculation results in tables.

Proposition 8.2. The space of algebraic restrictions of differential 2forms to $g$ is the 8-dimensional vector space spanned by the following algebraic restrictions:

$$
\begin{aligned}
& a_{8}=\left[d x_{1} \wedge d x_{2}\right]_{g}, \quad a_{10}=\left[d x_{3} \wedge d x_{1}\right]_{g}, \quad a_{11}=\left[x_{1} d x_{1} \wedge d x_{2}\right]_{g}, \\
& a_{12}=\left[d x_{2} \wedge d x_{3}\right]_{g}, \quad a_{13}=\left[x_{2} d x_{1} \wedge d x_{2}\right]_{g}, \quad a_{14}=\left[x_{1}^{2} d x_{1} \wedge d x_{2}\right]_{g} \text {, } \\
& a_{15}=\left[x_{3} d x_{1} \wedge d x_{2}\right]_{g}, \quad a_{16}=\left[x_{1} x_{2} d x_{1} \wedge d x_{2}\right]_{g},
\end{aligned}
$$

where $\delta$ is the quasi-degree of $a_{\delta}$.

Table 6. Quasi-homogeneous function-germs of quasidegree 10, 12, 14 vanishing on the curve $t \mapsto\left(t^{3}, t^{5}, t^{7}\right)$

\begin{tabular}{ccc}
\hline Quasi-degree $\delta$ & $h_{\delta}$ & Differential $d h_{\delta}$ \\
\hline 10 & $x_{1} x_{3}-x_{2}^{2}$ & $x_{1} d x_{3}+x_{3} d x_{1}-2 x_{2} d x_{2}$ \\
12 & $x_{2} x_{3}-x_{1}^{4}$ & $x_{2} d x_{3}+x_{3} d x_{2}-4 x_{1}^{3} d x_{1}$ \\
14 & $x_{1}^{3} x_{2}-x_{3}^{2}$ & $3 x_{1}^{2} x_{2} d x_{1}+x_{1}^{3} d x_{2}-2 x_{3} d x_{3}$ \\
\hline
\end{tabular}

Sketch of proof. We use the same method as in the previous section; see Tables 6 and 7. 
Table 7. The quasi-homogeneous basis of algebraic restrictions of 2-forms to the curve $t \mapsto\left(t^{3}, t^{5}, t^{7}\right)$

\begin{tabular}{|c|c|c|c|c|}
\hline$\delta$ & Basis & Forms & Relations & Proof \\
\hline 8 & $a_{8}$ & $\alpha_{8}=d x_{1} \wedge d x_{2}$ & $a_{8}:=\left[\alpha_{8}\right]_{g}$ & \\
\hline 10 & $a_{10}$ & $\alpha_{10}=d x_{3} \wedge d x_{1}$ & $a_{10}:=\left[\alpha_{10}\right]_{g}$ & \\
\hline 11 & $a_{11}$ & $x_{1} \alpha_{8}$ & $a_{11}:=x_{1} a_{8}$ & \\
\hline 12 & $a_{12}$ & $\alpha_{12}=d x_{2} \wedge d x_{3}$ & $a_{12}:=\left[\alpha_{12}\right]_{g}$ & \\
\hline 13 & $a_{13}$ & $\begin{array}{l}x_{2} \alpha_{8} \\
x_{1} \alpha_{10}\end{array}$ & $\begin{aligned} a_{13} & :=x_{2} a_{8} \\
x_{1} a_{10} & =-2 a_{13}\end{aligned}$ & {$\left[d h_{10} \wedge d x_{1}\right]_{g}=0$} \\
\hline 14 & $a_{14}$ & $x_{1}^{2} \alpha_{8}$ & $a_{14}:=x_{1}^{2} a_{8}$ & \\
\hline 15 & $a_{15}$ & $\begin{array}{l}x_{3} \alpha_{8} \\
x_{2} \alpha_{10} \\
x_{1} \alpha_{12} \\
\end{array}$ & $\begin{array}{l}a_{15}:=x_{3} a_{8} \\
x_{2} a_{10}=a_{15} \\
x_{1} a_{12}=a_{15}\end{array}$ & $\begin{array}{l}{\left[d h_{12} \wedge d x_{1}\right]_{g}=0} \\
{\left[d h_{10} \wedge d x_{2}\right]_{g}=0}\end{array}$ \\
\hline 16 & $a_{16}$ & $\begin{array}{c}x_{1} x_{2} \alpha_{8} \\
x_{1}^{2} \alpha_{10}\end{array}$ & $\begin{array}{l}a_{16}:=x_{1} x_{2} a_{8} \\
x_{1}^{2} a_{10}=-2 a_{16}\end{array}$ & $x_{1}\left[d h_{10} \wedge d x_{1}\right]_{g}=0$ \\
\hline 17 & 0 & $\begin{array}{l}x_{1}^{3} \alpha_{8} \\
x_{3} \alpha_{10} \\
x_{2} \alpha_{12}\end{array}$ & $\begin{array}{c}x_{1}^{3} a_{8}=0 \\
x_{3} a_{10}=0 \\
x_{2} a_{12}=0\end{array}$ & $\begin{array}{l}{\left[d h_{14} \wedge d x_{1}\right]_{g}=0} \\
{\left[d h_{12} \wedge d x_{2}\right]_{g}=0} \\
{\left[d h_{10} \wedge d x_{3}\right]_{g}=0}\end{array}$ \\
\hline 18 & 0 & $\begin{array}{c}x_{1} x_{3} \alpha_{8} \\
x_{1} x_{2} \alpha_{10} \\
x_{1}^{2} \alpha_{12}\end{array}$ & $\begin{array}{c}x_{1} x_{3} a_{8}=0 \\
x_{1} x_{2} a_{10}=0 \\
x_{1}^{2} a_{12}=0\end{array}$ & $\begin{array}{l}x_{1}\left[d h_{12} \wedge d x_{1}\right]_{g}=0 \\
x_{1}\left[d h_{10} \wedge d x_{2}\right]_{g}=0 \\
x_{2}\left[d h_{10} \wedge d x_{1}\right]_{g}=0\end{array}$ \\
\hline 19 & 0 & $\begin{array}{l}x_{1}^{2} x_{2} \alpha_{8} \\
x_{1}^{3} \alpha_{10} \\
x_{3} \alpha_{12}\end{array}$ & $\begin{array}{c}x_{1}^{2} x_{2} a_{8}=0 \\
x_{2}^{2} a_{10}=0 \\
x_{3} a_{12}=0\end{array}$ & $\begin{array}{c}x_{1}^{2}\left[d h_{10} \wedge d x_{1}\right]_{g}=0 \\
{\left[d h_{12} \wedge d x_{3}\right]_{g}=0} \\
{\left[d h_{14} \wedge d x_{2}\right]_{g}=0}\end{array}$ \\
\hline$\geq 20$ & 0 & $\begin{array}{l}x_{1} \beta_{\geq 17} \\
x_{2} \beta_{\geq 15} \\
x_{3} \beta_{\geq 13}\end{array}$ & $\begin{array}{c}b_{\geq 17}:=\left[\beta_{\geq 17}\right]_{g} \\
x_{1} b_{17}=0 \\
b_{\geq 15}:=\left[\beta_{\geq 15}\right]_{g} \\
x_{2} b_{\geq 15}=0 \\
b_{\geq 13}:=\left[\beta_{\geq 13}\right]_{g} \\
x_{3} b_{\geq 13}=0\end{array}$ & $\begin{array}{c}x_{2} b_{\geq 15}=x_{1} b_{\geq 17}^{\prime} \\
x_{3} b_{\geq 13}=x_{1} b_{\geq 17}^{\prime \prime} \\
\delta\left(b_{\geq 17}\right) \geq 17 \\
b_{\geq 17}=0\end{array}$ \\
\hline
\end{tabular}

Proposition 8.3. The space of algebraic restrictions of closed differential 2-forms to the image of $f$ is the 7-dimensional vector space spanned by the following algebraic restrictions:

$$
a_{8}, a_{10}, a_{11}, a_{12}, a_{13}, a_{14}, a_{16} .
$$

Proof. By Proposition 8.2 it is easy to see that $d a_{i}=0$ for $i \neq 15$ and $d a_{15} \neq 0$. By Proposition 3.9 we get the result. 
Proposition 8.4. Any algebraic restriction of a symplectic form to $f$ is diffeomorphic to one of the following: $\pm a_{8}, \pm a_{10}+c a_{11}, a_{11}+c a_{12}, a_{11}, \pm a_{12}$ $+c a_{13}, a_{13}+c a_{14}, \pm a_{14}, \pm a_{16}, 0$, where the parameter $c \in \mathbb{R}$ is a modulus.

Sketch of proof. The vector fields $X_{s}$ (see Theorem 6.12) are

$$
\begin{gathered}
X_{0}=E=3 x_{1} \frac{\partial}{\partial x_{1}}+5 x_{2} \frac{\partial}{\partial x_{2}}+7 x_{3} \frac{\partial}{\partial x_{3}}, \quad X_{2}=3 x_{2} \frac{\partial}{\partial x_{1}}+5 x_{3} \frac{\partial}{\partial x_{2}}+7 x_{1}^{3} \frac{\partial}{\partial x_{3}}, \\
X_{3}=x_{1} E, \quad X_{4}=3 x_{3} \frac{\partial}{\partial x_{1}}+5 x_{1}^{2} \frac{\partial}{\partial x_{2}}+7 x_{1}^{2} x_{2} \frac{\partial}{\partial x_{3}} \\
X_{5}=x_{2} E, \quad X_{6}=x_{1}^{2} E, \quad X_{7}=x_{3} E, \quad X_{8}=x_{1} x_{2} E .
\end{gathered}
$$

Their actions on the space of algebraic restrictions of closed 2-forms are given in Table 8 . From these data we obtain the classification of algebraic restrictions as in the previous section.

Table 8. Infinitesimal actions on algebraic restrictions of closed 2-forms to the curve $t \mapsto\left(t^{3}, t^{5}, t^{7}\right)$

\begin{tabular}{|c|ccccccc|}
\hline $\mathcal{L}_{X_{i}} a_{j}$ & $a_{8}$ & $a_{10}$ & $a_{11}$ & $a_{12}$ & $a_{13}$ & $a_{14}$ & $a_{16}$ \\
\hline$X_{0}=E$ & $8 a_{8}$ & $10 a_{10}$ & $11 a_{11}$ & $12 a_{12}$ & $13 a_{13}$ & $14 a_{14}$ & $16 a_{16}$ \\
$X_{2}$ & $-5 a_{10}$ & $-3 a_{12}$ & $13 a_{13}$ & $-21 a_{14}$ & 0 & $16 a_{16}$ & 0 \\
$X_{3}=x_{1} E$ & $11 a_{11}$ & $-26 a_{13}$ & $14 a_{14}$ & 0 & $16 a_{16}$ & 0 & 0 \\
$X_{4}$ & $-3 a_{12}$ & $-7 a_{14}$ & 0 & $6 a_{16}$ & 0 & 0 & 0 \\
$X_{5}=x_{2} E$ & $13 a_{13}$ & 0 & $16 a_{16}$ & 0 & 0 & 0 & 0 \\
$X_{6}=x_{1}^{2} E$ & $11 a_{14}$ & $-32 a_{16}$ & 0 & 0 & 0 & 0 & 0 \\
$X_{7}=x_{3} E$ & 0 & 0 & 0 & 0 & 0 & 0 & 0 \\
$X_{8}=x_{1} x_{2} E$ & $4 a_{16}$ & 0 & 0 & 0 & 0 & 0 & 0 \\
\hline
\end{tabular}

From Table 8 and Theorem 6.12 we also see that the tangent space to the orbit of $\pm a_{10}+c a_{11}$ at $\pm a_{10}+c a_{11}$ is spanned by $\pm 10 a_{10}+11 c a_{11}, a_{12}, a_{13}$, $a_{14}, a_{16} ; a_{11}$ does not belong to it. Therefore the parameter $c$ is a modulus in the normal form $\pm a_{10}+c a_{11}$.

In the same way we prove that $c$ is a modulus in the other normal forms.

9. Symplectic singularities of curves with semigroup $(3,7,8)$. This section presents a symplectic classification of curves with semigroup $(3,7,8)$.

Theorem 9.1. Let $\left(\mathbb{R}^{2 n}, \omega_{0}=\sum_{i=1}^{n} d p_{i} \wedge d q_{i}\right)$ be the symplectic space with the canonical coordinates $\left(p_{1}, q_{1}, \ldots, p_{n}, q_{n}\right)$. Then the germ of a curve $f:(\mathbb{R}, 0) \rightarrow\left(\mathbb{R}^{2 n}, 0\right)$ with semigroup $(3,7,8)$ is symplectically equivalent to one and only one of the curves in the second column of Table 9 for $n>2$, and $f$ is symplectically equivalent to one and only one of the curves in the second column and rows $1-3,5$ and 7 for $n=2$. The parameters $c, c_{1}, c_{2}$ are moduli. 
The symplectic multiplicity, the index of isotropy and the Lagrangian tangency order are given in the third, fourth and fifth columns of Table 9.

Table 9. Symplectic classification of curves with semigroup $(3,7,8)$

\begin{tabular}{llccc}
\hline & Normal form of $f$ & $\mu_{\text {sympl }}(f)$ & $\iota(f)$ & $\operatorname{Lt}(f)$ \\
\hline 1 & $t \mapsto\left(t^{3}, \pm t^{7}, t^{8}, c t^{3}, 0, \ldots, 0\right)$ & 1 & 0 & 7 \\
2 & $t \mapsto\left(t^{3}, t^{8}, t^{7}, c t^{6}, 0, \ldots, 0\right)$ & 2 & 0 & 8 \\
3 & $t \mapsto\left(t^{3}, t^{10}+c_{1} t^{11}, t^{7}, c_{2} t^{8}, 0, \ldots, 0\right), c_{2} \neq 0$ & 4 & 0 & 8 \\
4 & $t \mapsto\left(t^{3}, t^{10}, t^{8}, c t^{6}, t^{7}, 0, \ldots, 0\right)$ & 4 & 1 & 10 \\
5 & $t \mapsto\left(t^{3}, \pm t^{11}+c_{2} t^{13}, t^{7}, c_{1} t^{8}, 0, \ldots, 0\right), c_{1} \neq 0$ & 5 & 0 & 8 \\
6 & $t \mapsto\left(t^{3}, \pm t^{11}, t^{7}, c t^{9}, t^{8}, 0, \ldots, 0\right)$ & 5 & 1 & 11 \\
7 & $t \mapsto\left(t^{3}, c_{1} t^{13}+c_{2} t^{14}, t^{7}, t^{8}, 0, \ldots, 0\right)$ & 6 & 0 & 8 \\
8 & $t \mapsto\left(t^{3}, \pm t^{13}, t^{7}, c_{1} t^{10}, t^{8}, c_{2} t^{10}, 0, \ldots, 0\right), c_{2} \neq 0$ & 7 & 1 & 11 \\
9 & $t \mapsto\left(t^{3}, \pm t^{13}, t^{7}, c t^{10}, t^{8}, 0, \ldots, 0\right)$ & 7 & 2 & 13 \\
10 & $t \mapsto\left(t^{3}, t^{14}, t^{7}, c_{1} t^{11}, t^{8}, c_{2} t^{11}, 0, \ldots, 0\right), c_{1} \neq 0$ & 8 & 1 & 11 \\
11 & $t \mapsto\left(t^{3}, t^{14}, t^{7}, 0, t^{8}, c t^{11}, 0, \ldots, 0\right)$ & 8 & 2 & 14 \\
12 & $t \mapsto\left(t^{3}, c_{1} t^{17}, t^{7}, \pm t^{11}, t^{8}, c_{2} t^{11}, 0, \ldots, 0\right)$ & 9 & 1 & 11 \\
13 & $t \mapsto\left(t^{3}, t^{16}, t^{7}, c t^{13}, t^{8}, 0, \ldots, 0\right)$ & 9 & 3 & 16 \\
14 & $t \mapsto\left(t^{3}, \pm t^{17}, t^{7}, 0, t^{8}, 0, \ldots, 0\right)$ & 9 & 3 & 17 \\
15 & $t \mapsto\left(t^{3}, 0, t^{7}, 0, t^{8}, 0, \ldots, 0\right)$ & 10 & $\infty$ & $\infty$ \\
\hline
\end{tabular}

Let $f: \mathbb{R} \ni t \mapsto f(t) \in \mathbb{R}^{2 n}$ be the germ of a smooth or $\mathbb{R}$-analytic curve $\mathcal{A}$-equivalent to $t \mapsto\left(t^{3}, t^{7}, t^{8}, 0, \ldots, 0\right)$. First we calculate the space of algebraic restrictions of 2 -forms to the image of $f$ in $\mathbb{R}^{2 n}$.

Table 10. Quasi-homogeneous function-germs of quasidegree 14, 15, 16 vanishing on the curve $t \mapsto\left(t^{3}, t^{7}, t^{8}\right)$

\begin{tabular}{ccc}
\hline Quasi-degree $\delta$ & $h_{\delta}$ & Differential $d h_{\delta}$ \\
\hline 14 & $x_{1}^{2} x_{3}-x_{2}^{2}$ & $2 x_{1} x_{3} d x_{1}+x_{1}^{2} d x_{3}-2 x_{2} d x_{2}$ \\
15 & $x_{2} x_{3}-x_{1}^{5}$ & $x_{2} d x_{3}+x_{3} d x_{2}-5 x_{1}^{4} d x_{1}$ \\
16 & $x_{1}^{3} x_{2}-x_{3}^{2}$ & $3 x_{1}^{2} x_{2} d x_{1}+x_{1}^{3} d x_{2}-2 x_{3} d x_{3}$ \\
\hline
\end{tabular}

Proposition 9.2. The space of algebraic restrictions of differential 2forms to $g$ is the 12-dimensional vector space spanned by the following algebraic restrictions:

$$
\begin{aligned}
& a_{10}=\left[d x_{1} \wedge d x_{2}\right]_{g}, \quad a_{11}=\left[d x_{3} \wedge d x_{1}\right]_{g}, \quad a_{13}=\left[x_{1} d x_{1} \wedge d x_{2}\right]_{g}, \\
& a_{14}=\left[x_{1} d x_{3} \wedge d x_{1}\right]_{g}, \quad a_{15}=\left[d x_{2} \wedge d x_{3}\right]_{g}, \quad a_{16}=\left[x_{1}^{2} d x_{1} \wedge d x_{2}\right]_{g}, \\
& a_{17}=\left[x_{2} d x_{1} \wedge d x_{2}\right]_{g}, \quad a_{18}^{+}=\left[x_{1} d x_{2} \wedge d x_{3}\right]_{g}, \quad a_{18}^{-}=\left[x_{2} d x_{3} \wedge d x_{1}\right]_{g}, \\
& a_{19}=\left[x_{3} d x_{3} \wedge d x_{1}\right]_{g}, \quad a_{20}=\left[x_{1} x_{2} d x_{1} \wedge d x_{2}\right]_{g}, \quad a_{21}=\left[x_{1} x_{3} d x_{1} \wedge d x_{2}\right]_{g}, \\
& \text { where } \delta \text { is the quasi-degree of } a_{\delta} \text {. }
\end{aligned}
$$


Sketch of proof. We use the same method as in the previous sections; see Tables 10 and 11.

Table 11. The quasi-homogeneous basis of algebraic restrictions of 2 -forms to the curve $t \mapsto\left(t^{3}, t^{7}, t^{8}\right)$

\begin{tabular}{|c|c|c|c|c|}
\hline$\delta$ & Basis & Forms & Relations & Proof \\
\hline 10 & $a_{10}$ & $\alpha_{10}=d x_{1} \wedge d x_{2}$ & $a_{10}:=\left[\alpha_{10}\right]_{g}$ & \\
\hline 11 & $a_{11}$ & $\alpha_{11}=d x_{3} \wedge d x_{1}$ & $a_{11}:=\left[\alpha_{11}\right]_{g}$ & \\
\hline 13 & $a_{13}$ & $x_{1} \alpha_{10}$ & $a_{13}:=x_{1} a_{10}$ & \\
\hline 14 & $a_{14}$ & $\alpha_{14}=x_{1} \alpha_{11}$ & $a_{14}:=x_{1} a_{11}$ & \\
\hline 15 & $a_{15}$ & $\alpha_{15}=d x_{2} \wedge d x_{3}$ & $a_{15}:=\left[\alpha_{15}\right]_{g}$ & \\
\hline 16 & $a_{16}$ & $x_{1}^{2} \alpha_{10}$ & $a_{16}:=x_{1}^{2} a_{10}$ & \\
\hline 17 & $a_{17}$ & $\begin{array}{l}x_{2} \alpha_{10} \\
x_{1}^{2} \alpha_{11}\end{array}$ & $\begin{array}{c}a_{17}:=x_{2} a_{10} \\
x_{1}^{2} a_{11}=-2 a_{17}\end{array}$ & {$\left[d h_{14} \wedge d x_{1}\right]_{g}=0$} \\
\hline 18 & $\begin{array}{l}a_{18}^{+} \\
a_{18}^{-}\end{array}$ & $\begin{array}{l}x_{1} \alpha_{15} \\
x_{2} \alpha_{11} \\
x_{3} \alpha_{10} \\
\end{array}$ & $\begin{array}{l}a_{18}^{+}:=x_{1} a_{15} \\
a_{18}^{-}:=x_{2} a_{11} \\
x_{3} a_{10}=a_{18}^{-}\end{array}$ & {$\left[d h_{15} \wedge d x_{1}\right]_{g}=0$} \\
\hline 19 & $a_{19}$ & $\begin{array}{l}x_{3} \alpha_{11}, \\
x_{1}^{3} \alpha_{10}\end{array}$ & $\begin{array}{c}a_{19}:=x_{3} a_{11} \\
x_{1}^{3} a_{10}=-2 a_{19}\end{array}$ & {$\left[d h_{16} \wedge d x_{1}\right]_{g}=0$} \\
\hline 20 & $a_{20}$ & $\begin{array}{c}x_{1} x_{2} \alpha_{10}, \\
x_{1}^{3} \alpha_{11} \\
\end{array}$ & $\begin{array}{l}a_{20}:=x_{1} x_{2} a_{10} \\
x_{1}^{3} a_{10}=-2 a_{20}\end{array}$ & $x_{1}\left[d h_{14} \wedge d x_{1}\right]_{g}=0$ \\
\hline 21 & $a_{21}$ & $\begin{array}{c}x_{1} x_{3} \alpha_{10}, \\
x_{1} x_{2} \alpha_{11} \\
x_{1}^{2} \alpha_{15} \\
\end{array}$ & $\begin{array}{c}a_{21}:=x_{1} x_{3} a_{10} \\
x_{1} x_{2} a_{11}=a_{21} \\
x_{1}^{2} a_{15}=2 a_{21} \\
\end{array}$ & $\begin{array}{c}{\left[d h_{14} \wedge d x_{2}\right]_{g}=0} \\
x_{1}\left[d h_{15} \wedge d x_{1}\right]_{g}=0\end{array}$ \\
\hline 22 & 0 & $\begin{array}{c}x_{1}^{4} \alpha_{10}, \\
x_{1} x_{3} \alpha_{11} \\
x_{2} \alpha_{15}\end{array}$ & $\begin{array}{c}x_{1}^{4} a_{10}=0 \\
x_{1} x_{3} a_{11}=0 \\
x_{2} a_{15}=0\end{array}$ & $\begin{array}{c}x_{1}\left[d h_{16} \wedge d x_{1}\right]_{g}=0 \\
{\left[d h_{15} \wedge d x_{2}\right]_{g}=0} \\
{\left[d h_{14} \wedge d x_{3}\right]_{g}=0}\end{array}$ \\
\hline 23 & 0 & $\begin{array}{c}x_{1}^{2} x_{2} \alpha_{10}, \\
x_{1}^{4} \alpha_{11}, \\
x_{3} \alpha_{15} \\
\end{array}$ & $\begin{array}{c}x_{1}^{2} x_{2} a_{10}=0 \\
x_{1}^{4} a_{11}=0 \\
x_{3} a_{15}=0\end{array}$ & $\begin{array}{c}x_{1}^{2}\left[d h_{14} \wedge d x_{1}\right]_{g}=0 \\
{\left[d h_{16} \wedge d x_{2}\right]_{g}=0} \\
{\left[d h_{15} \wedge d x_{3}\right]_{g}=0}\end{array}$ \\
\hline 24 & 0 & $\begin{array}{c}x_{2}^{2} \alpha_{10}, \\
x_{1}^{2} x_{2} \alpha_{11} \\
x_{3} \alpha_{15}\end{array}$ & $\begin{array}{c}x_{2}^{2} a_{10}=0 \\
x_{1}^{2} x_{2} a_{11}=0 \\
x_{3} a_{15}=0\end{array}$ & $\begin{array}{c}x_{1}^{2}\left[d h_{15} \wedge d x_{1}\right]_{g}=0 \\
{\left[d h_{16} \wedge d x_{3}\right]_{g}=0} \\
x_{1}\left[d h_{14} \wedge d x_{2}\right]_{g}=0\end{array}$ \\
\hline$\geq 25$ & 0 & $\begin{array}{l}x_{1} \beta_{\geq 22}, \\
x_{2} \beta_{\geq 18}, \\
x_{3} \beta_{\geq 17}\end{array}$ & $\begin{array}{c}b_{\geq 22}:=\left[\beta_{\geq 22}\right]_{g} \\
x_{1} b_{\geq 22}=0 \\
b_{\geq 18}=:\left[\beta_{\geq 18}\right]_{g} \\
x_{2} b \geq 18=0 \\
b_{\geq 17}:=\left[\beta_{\geq 17}\right]_{g} \\
x_{3} b_{\geq 17}=0\end{array}$ & $\begin{array}{c}x_{2} b_{\geq 18}=x_{1} b_{\geq 22}^{\prime} \\
x_{3} b_{\geq 17}=x_{1} b_{\geq 22}^{\prime \prime} \\
\delta\left(b_{\geq 22}\right) \geq 22 \\
b_{\geq 22}=0\end{array}$ \\
\hline
\end{tabular}


Proposition 9.3. The space of algebraic restrictions of closed differential 2-forms to the image of $g$ is the 10-dimensional vector space spanned by the following algebraic restrictions:

$$
a_{10}, a_{11}, a_{13}, a_{14}, a_{15}, a_{16}, a_{17}, a_{18}=a_{18}^{+}-a_{18}^{-}, a_{19}, a_{20} .
$$

Proof. It is easy to see that $d a_{i}=0$ for $i \neq 18,21, d a_{18}^{+}=d a_{18}^{-} \neq 0$ and $d a_{21} \neq 0$. Hence the algebraic restriction $a_{18}^{+}-a_{18}^{-}$is closed and $d a_{18}^{-}, d a_{21}$ are linearly independent. Thus Theorem 3.9 implies the result.

Proposition 9.4. Any algebraic restriction of a symplectic form to $f$ is diffeomorphic to one of the following: $\pm a_{10}+c a_{11}, a_{11}+c a_{13}, a_{13}+c_{1} a_{14}+$ $c_{2} a_{15}, \pm a_{14}+c_{1} a_{15}+c_{2} a_{16}, a_{15}+c_{1} a_{16}+c_{2} a_{17}, \pm a_{16}+c_{1} a_{17}+c_{2} a_{18}, a_{17}+$ $c_{1} a_{18}+c_{2} a_{19}, \pm a_{18}+c_{1} a_{19}+c_{2} a_{20}, a_{19}+c a_{20}, \pm a_{20}, 0$, where $c, c_{1}, c_{2} \in \mathbb{R}$.

The parameters $c, c_{1}, c_{2}$ are moduli.

Sketch of proof. The vector fields $X_{s}$ (see Theorem 6.12) are

$$
\begin{gathered}
X_{0}=E=3 x_{1} \frac{\partial}{\partial x_{1}}+7 x_{2} \frac{\partial}{\partial x_{2}}+8 x_{3} \frac{\partial}{\partial x_{3}}, \quad X_{3}=x_{1} E \\
X_{4}=3 x_{2} \frac{\partial}{\partial x_{1}}+7 x_{1} x_{3} \frac{\partial}{\partial x_{2}}+8 x_{1}^{4} \frac{\partial}{\partial x_{3}}, \quad X_{5}=3 x_{3} \frac{\partial}{\partial x_{1}}+7 x_{1}^{4} \frac{\partial}{\partial x_{2}}+8 x_{1}^{2} x_{2} \frac{\partial}{\partial x_{3}}, \\
X_{6}=x_{1}^{2} E, \quad X_{7}=x_{2} E, \quad X_{8}=x_{3} E, \quad X_{9}=x_{1}^{3} E, \quad X_{10}=x_{1} x_{2} E .
\end{gathered}
$$

Their actions on the space of algebraic restrictions of closed 2-forms are shown in Table 12. From these data we obtain a classification of the algebraic restrictions as in the previous section.

Table 12. Infinitesimal actions on algebraic restrictions of closed 2 -forms to the curve $t \mapsto\left(t^{3}, t^{7}, t^{8}\right)$

\begin{tabular}{|c|cccccccc|}
\hline $\mathcal{L}_{X_{i}} a_{j}$ & $a_{10}$ & $a_{11}$ & $a_{13}$ & $a_{14}$ & $a_{15}$ & $a_{16}$ & $a_{17}$ & $a_{\delta \geq 18}$ \\
\hline$E$ & $10 a_{10}$ & $11 a_{11}$ & $13 a_{13}$ & $14 a_{14}$ & $15 a_{15}$ & $16 a_{16}$ & $17 a_{17}$ & $\delta a_{\delta}$ \\
$X_{3}$ & $13 a_{13}$ & $14 a_{14}$ & $16 a_{16}$ & $17 a_{17}$ & $15 a_{18}$ & $-38 a_{19}$ & $20 a_{20}$ & 0 \\
$X_{4}$ & $-7 a_{14}$ & $-3 a_{15}$ & $17 a_{17}$ & $-3 a_{18}$ & $9 a_{19}$ & $20 a_{20}$ & 0 & 0 \\
$X_{5}$ & $-3 a_{15}$ & $-8 a_{16}$ & $-3 a_{18}$ & $19 a_{19}$ & $12 a_{20}$ & 0 & 0 & 0 \\
$X_{6}$ & $16 a_{16}$ & $17 a_{17}$ & $-38 a_{19}$ & $-38 a_{20}$ & 0 & 0 & 0 & 0 \\
$X_{7}$ & $17 a_{17}$ & $-3 a_{18}$ & $20 a_{20}$ & 0 & 0 & 0 & 0 & 0 \\
$X_{8}$ & $-3 a_{18}$ & $19 a_{19}$ & 0 & 0 & 0 & 0 & 0 & 0 \\
$X_{9}$ & $-38 a_{19}$ & $-40 a_{20}$ & 0 & 0 & 0 & 0 & 0 & 0 \\
$X_{10}$ & $20 a_{20}$ & 0 & 0 & 0 & 0 & 0 & 0 & 0 \\
\hline
\end{tabular}

Now we prove that the parameters $c, c_{1}, c_{2}$ are moduli in the normal forms. The proofs are very similar in all cases. As an example we consider the normal form $a_{13}+c_{1} a_{14}+c_{2} a_{15}$, the first normal form with two parameters. From Table 12 and Theorem 6.12 we see that the tangent space to the 
orbit of $a_{13}+c_{1} a_{14}+c_{2} a_{15}$ at $a_{13}+c_{1} a_{14}+c_{2} a_{15}$ is spanned by the linearly independent algebraic restrictions $a_{13}+14 c_{1} a_{14}+15 c_{2} a_{15}, a_{16}, a_{17}, a_{18}$, $a_{19}, a_{20}$. Hence the algebraic restrictions $a_{14}$ and $a_{15}$ do not belong to it. Therefore the parameters $c_{1}$ and $c_{2}$ are independent moduli in the normal form $a_{13}+c_{1} a_{14}+c_{2} a_{15}$.

Acknowledgements. The author wishes to express his thanks to M. Zhitomirskii for suggesting the subject and for many helpful conversations and remarks during the writing of this paper. The author thanks Z. Jelonek for very useful remarks on the proof of Theorem 2 and the referee for many valuable suggestions.

The work of the author was supported by Institute of Mathematics, Polish Academy of Sciences and by the European Union in the framework of European Social Fund through the Warsaw University of Technology Development Programme.

\section{References}

[A1] V. I. Arnold, First steps of local symplectic algebra, in: Differential Topology, Infinite-Dimensional Lie Algebras, and Applications, D. B. Fuchs' 60th anniversary collection, Amer. Math. Soc. Transl. 194, Amer. Math. Soc., Providence, RI, 1999, 1-8.

[A2] - Simple singularities of curves, Proc. Steklov Inst. Math. 226 (1999), 20-28.

[AG] V. I. Arnold and A. B. Givental, Symplectic geometry, in: Dynamical Systems, IV, Encyclopaedia Math. Sci. 4, Springer, Berlin, 2001, 1-138.

[AVG] V. I. Arnold, S. M. Gusein-Zade and A. N. Varchenko, Singularities of Differentiable Maps, Vol. 1, Birkhäuser, Boston, 1985.

[BH] T. Bloom and M. Herrera, De Rham cohomology of an analytic space, Invent. Math. 7 (1969), 275-296.

[BG] J. W. Bruce and T. J. Gaffney, Simple singularities of mappings $(\mathbb{C}, 0) \rightarrow\left(\mathbb{C}^{2}, 0\right)$, J. London Math. Soc. (2) 26 (1982), 465-474.

[BPW] J. W. Bruce, A. A. du Plessis and L. C. Wilson, Discriminants and liftable vector fields, J. Algebraic Geom. 3 (1994), 725-753.

[DJZ1] W. Domitrz, S. Janeczko and M. Zhitomirskii, Relative Poincaré lemma, contractibility, quasi-homogeneity and vector fields tangent to a singular variety, Illinois J. Math. 48 (2004), 803-835.

[DJZ2] - - - - Symplectic singularities of varietes: the method of algebraic restrictions, J. Reine Angew. Math. 618 (2008), 197-235.

[DR] W. Domitrz and J. H. Rieger, Volume preserving subgroups of $\mathcal{A}$ and $\mathcal{K}$ and singularities in unimodular geometry, Math. Ann., to appear.

[GH] C. G. Gibson and C. A. Hobbs, Simple singularities of space curves, Math. Proc. Cambridge Philos. Soc. 113 (1993), 297-310.

[IJ1] G. Ishikawa and S. Janeczko, Symplectic bifurcations of plane curves and isotropic liftings, Quart. J. Math. 54 (2003), 73-102.

[IJ2] - - - Symplectic singularities of isotropic mappings, in: Geometric Singularity Theory, Banach Center Publ. 65, Inst. Math., Polish Acad. Sci., 2004, 85-106. 
[K] P. A. Kolgushkin, Classification of simple multigerms of curves in a space endowed with a symplectic structure, St. Petersburg Math. J. 15 (2004), 103-126.

[R] J. L. Ramírez Alfonsín, The Diophantine Frobenius Problem, Oxford Lecture Ser. Math. Appl. 30, Oxford Univ. Press, Oxford, 2005.

[S] J. J. Sylvester, Question 7382, Mathematical Questions from the Educational Times 37: 26 (1884).

[W] C. T. C. Wall, Singular Points of Plane Curves, London Math. Soc. Student Texts 63, Cambridge Univ. Press, Cambridge, 2004.

[Za] V. M. Zakalyukin, Rearrangements of wave fronts that depend on a certain parameter, Funktsional. Anal. i Prilozhen. 10 (1976), no. 2, 69-70 (in Russian).

[Z] M. Zhitomirskii, Relative Darboux theorem for singular manifolds and local contact algebra, Canad. J. Math. 57 (2005), 1314-1340.

Faculty of Mathematics and Information Science

Warsaw University of Technology

Plac Politechniki 1

00-661 Warszawa, Poland

E-mail: domitrz@mini.pw.edu.pl

and

Institute of Mathematics

Polish Academy of Sciences

Śniadeckich 8

P.O. Box 21

00-956 Warszawa, Poland

Received 5 June 2008;

in revised form 27 February 2009 